\title{
Tensor products for Gelfand-Shilov and Pilipović distribution spaces
}

\section{Joachim Toft $^{1}$}

Received: 30 August 2018/Accepted: 5 October 2019/Published online: 31 October 2019

(C) The Author(s) 2019

\section{Abstract}

We show basic properties on tensor products for Gelfand-Shilov distributions and Pilipović distributions. This also includes the Fubini's property of such tensor products. We also apply the Fubini property to deduce some properties for shorttime Fourier transforms of Gelfand-Shilov and Pilipović distributions.

Keywords Ultradistributions · Fubini · Fourier transform · Bargmann transform $\cdot$ Gelfand tripple

Mathematics Subject Classification 46A32 - 46Fxx $\cdot 46 \mathrm{M} 05$

\section{Introduction}

An important issue in mathematics concerns tensor products. When considering the functions $f_{j}$ defined on $\Omega_{j} \subseteq \mathbf{R}^{d_{j}}, j=1,2$, and with values in $\mathbf{C}$, their tensor product $f_{1} \otimes f_{2}$ is the function from $\Omega_{1} \times \Omega_{2}$ to $\mathbf{C}$ given by the formula

$$
\left(f_{1} \otimes f_{2}\right)\left(x_{1}, x_{2}\right)=f_{1}\left(x_{1}\right) f_{2}\left(x_{2}\right), \quad x_{j} \in \Omega_{j}, j=1,2 .
$$

Let $f_{j}, \varphi_{j} \in \mathscr{S}\left(\mathbf{R}^{d_{j}}\right), f=f_{1} \otimes f_{2}, \varphi \in \mathscr{S}\left(\mathbf{R}^{d_{1}+d_{2}}\right)$, and let $\psi_{1}$ and $\psi_{2}$ be given by

$$
\psi_{1}\left(x_{1}\right)=\left\langle f_{2}, \varphi\left(x_{1}, \cdot\right)\right\rangle \quad \text { and } \quad \psi_{2}\left(x_{2}\right)=\left\langle f_{1}, \varphi\left(\cdot, x_{2}\right)\right\rangle
$$

(For notations, see. [9] and Sect. 2.) Then it follows that

$$
\left\langle f, \varphi_{1} \otimes \varphi_{2}\right\rangle=\left\langle f_{1}, \varphi_{1}\right\rangle\left\langle f_{2}, \varphi_{2}\right\rangle,
$$

and that the Fubini's property

Joachim Toft

joachim.toft@1nu.se

1 Department of Mathematics, Linnæus University, Växjö, Sweden 


$$
\langle f, \varphi\rangle=\left\langle f_{1}, \psi_{1}\right\rangle=\left\langle f_{2}, \psi_{2}\right\rangle
$$

holds.

The formula (1.2) and (1.3) are essential when searching for extensions of tensor products to distributions. By the analysis in [9, Chapter V and VII], we have the following.

Theorem 1.1 Let $f_{j} \in \mathscr{S}^{\prime}\left(\mathbf{R}^{d_{j}}\right), \varphi \in \mathscr{S}\left(\mathbf{R}^{d_{1}+d_{2}}\right)$ and let $\psi_{j}$ be given by (1.1), $j=1$, 2. Then $\psi_{j} \in \mathscr{S}\left(\mathbf{R}^{d_{2}}\right), j=1,2$, and there is a unique $f \in \mathscr{S}^{\prime}\left(\mathbf{R}^{d_{1}+d_{2}}\right)$ such that for every $\varphi_{1} \in \mathscr{S}\left(\mathbf{R}^{d_{1}}\right)$ and $\varphi_{2} \in \mathscr{S}\left(\mathbf{R}^{d_{2}}\right)$, (1.2) and (1.3) hold.

The existence of a distribution $f$ in the previous theorem which satisfies (1.2) can also be deduced by a general and abstract result on tensor products for nuclear spaces (see, [17, Chapter 50]). On the other hand, in order to reach the Fubini property (1.3), it seems that more structures are needed.

A more specific approach in the lines of the ideas in [17] is indicated in [10,14], where $\mathscr{S}\left(\mathbf{R}^{d}\right)$ and $\mathscr{S}^{\prime}\left(\mathbf{R}^{d}\right)$ are described by suitable series expansions of Hermite functions. By following such approaches, the situations are essentially reduced to questions on tensor products of weighted $\ell^{2}$ spaces, and both properties (1.2) and (1.3) follows from such approach.

In Sects. 3 and 4 we show that Theorem 1.1 holds in the context of GelfandShilov spaces, Pilipović spaces and their distribution (dual) spaces. In particular, we prove that the following results hold true.

Theorem 1.2 Let $s_{j}, \sigma_{j}>0, f_{j} \in\left(\mathcal{S}_{s_{j}}^{\sigma_{j}}\right)^{\prime}\left(\mathbf{R}^{d_{j}}\right), \varphi \in \mathcal{S}_{s_{1}, s_{2}}^{\sigma_{1}}\left(\mathbf{R}^{d_{1}+d_{2}}\right)$ and let $\psi_{j}$ be given by (1.1), $j=1,2$. Then $\psi_{j} \in \mathcal{S}_{s_{j}}^{\sigma_{j}}\left(\mathbf{R}^{d_{2}}\right), j=1,2$, and there is a unique $f \in$ $\left(\mathcal{S}_{s_{1}, s_{2}}^{\sigma_{1}}\right)^{\prime}\left(\mathbf{R}^{d_{1}+d_{2}}\right)$ such that for every $\varphi_{1} \in \mathcal{S}_{s_{1}}^{\sigma_{1}}\left(\mathbf{R}^{d_{1}}\right)$ and $\varphi_{2} \in \mathcal{S}_{s_{2}}^{\sigma_{2}}\left(\mathbf{R}^{d_{2}}\right)$, (1.2) and (1.3) hold.

The same holds true with $\Sigma_{s_{j}}^{\sigma_{j}},\left(\Sigma_{s_{j}}^{\sigma_{j}}\right)^{\prime}, \Sigma_{s_{1}, s_{2}}^{\sigma_{1}, \sigma_{2}}$ and $\left(\Sigma_{s_{1}, s_{2}}^{\sigma_{1}}\right)^{\prime}$ in place of $\Sigma_{s_{j}}^{\sigma_{j}},\left(\mathcal{S}_{s_{j}}^{\sigma_{j}}\right)^{\prime}$, $\mathcal{S}_{s_{1}, s_{2}}^{\sigma_{1}, \sigma_{2}}$ and $\left(\mathcal{S}_{s_{1}, s_{2}}^{\sigma_{1}, \sigma_{2}}\right)^{\prime}$, respectively, at each occurrence.

Theorem 1.3 Let $s \in \overline{\mathbf{R}_{b}}, f_{j} \in \mathcal{H}_{s}^{\prime}\left(\mathbf{R}^{d_{j}}\right), \varphi \in \mathcal{H}_{s}\left(\mathbf{R}^{d_{1}+d_{2}}\right)$ and let $\psi_{j}$ be given by (1.1), $j=1,2$. Then $\psi_{j} \in \mathcal{H}_{s}\left(\mathbf{R}^{d_{2}}\right), j=1,2$, and there is a unique $f \in \mathcal{H}_{s}^{\prime}\left(\mathbf{R}^{d_{1}+d_{2}}\right)$ such that for every $\varphi_{1} \in \mathcal{H}_{s}\left(\mathbf{R}^{d_{1}}\right)$ and $\varphi_{2} \in \mathcal{H}_{s}\left(\mathbf{R}^{d_{2}}\right)$, (1.2) and (1.3) hold.

The same holds true with $\mathcal{H}_{0, s}$ and $\mathcal{H}_{0, s}^{\prime}$ in place of $\mathcal{H}_{s}$ and $\mathcal{H}_{s}^{\prime}$, respectively, at each occurrence.

The distribution $f$ in Theorems 1.1, 1.2 or in Theorem 1.3 is called the tensor product of $f_{1}$ and $f_{2}$ and is denoted by $f_{1} \otimes f_{2}$ as before. We notice that in some cases, Theorem 1.2 is deduced in [4] (cf. [4, Appendix]).

We remark that Gelfand-Shilov spaces of functions and distributions appear naturally when discussing analyticity and well-posedness of solutions to partial differential equations (cf. [2, 3]). Pilipović spaces of functions and distributions often agree with Fourier-invariant Gelfand-Shilov spaces, and possess convenient mapping properties with respect to the Bargmann transform. They therefore seems 
to be suitable to have in background on problems in partial differential equations which have been transformed by the Bargmann transform (see, $[6,16]$ for more details).

Since the spaces in Theorems 1.2 and 1.3 are unions and intersections of nuclear spaces, the existence of $f$ satisfying (1.2) may be deduced by the abstract analogous results in [17]. Some parts of Theorem 1.2 are also proved in [10].

In Sect. 3 we give a proof of Theorem 1.2, by using the framework in [9] for the proof of Theorem 1.1. In Sect. 4 we use that Pilipović spaces and their distribution spaces can be described by unions and intersections of Hilbert spaces of Hermite series expansions. In similar ways as in [14], this essentially reduce the situation to deal with questions on tensor products of weighted $\ell^{2}$ spaces.

In the end of Sect. 3 we also give examples on how to apply the Fubini property (1.3) to deduce certain relations for short-time Fourier transforms (which is often called coherent state trasnforms in physics) of Gelfand-Shilov distributions (see, Example 3.7). In Sect. 4 we also discuss such questions for Pilipović spaces which are not Gelfand-Shilov distributions (cf. Remark 4.4).

\section{Preliminaries}

In this section we recall some basic facts. We start by giving the definition of Gelfand-Shilov spaces. Thereafter we recall the definition of Pilipović spaces and some of their properties.

\subsection{Gelfand-Shilov spaces}

We start by recalling some facts about Gelfand-Shilov spaces (cf. [5, 8]). Let $0<h, s_{j}, \sigma_{j} \in \mathbf{R}, j=1, \ldots, n$, be fixed, $d=d_{1}+\cdots+d_{n}$, where $d_{j} \geq 0$ are integers, and let

$$
\boldsymbol{s}=\left(s_{1}, \ldots, s_{n}\right) \in \mathbf{R}_{+}^{n} \quad \text { and } \quad \boldsymbol{\sigma}=\left(\sigma_{1}, \ldots, \sigma_{n}\right) \in \mathbf{R}_{+}^{n} .
$$

For multi-indices of multi-indices we let

$$
\begin{aligned}
& \alpha !^{s}=\alpha_{1} !^{s_{1}} \cdots \alpha_{n} !^{s_{n}}, \quad \quad x^{\alpha}=x^{\alpha_{1}} \cdots x^{\alpha_{n}}, \\
& D_{x}^{\alpha}=D_{x_{1}}^{\alpha_{1}} \cdots D_{x_{n}}^{\alpha_{n}} \quad \text { and } \quad|\alpha|=\left|\alpha_{1}\right|+\cdots+\left|\alpha_{n}\right|
\end{aligned}
$$

when

$$
\alpha=\left(\alpha_{1}, \ldots, \alpha_{n}\right) \in \mathbf{N}^{d_{1}} \times \cdots \times \mathbf{N}^{d_{n}}, \quad \text { and } \quad x=\left(x_{1}, \ldots, x_{n}\right) \in \mathbf{R}^{d_{1}} \times \cdots \times \mathbf{R}^{d_{n}} .
$$

For any $f \in C^{\infty}\left(\mathbf{R}^{d}\right)$, we let

$$
\|f\|_{\mathcal{S}_{s ; h}^{\sigma}} \equiv \sup \left(\frac{\left\|x^{\alpha} \partial_{x}^{\beta} f\right\|_{L^{\infty}\left(\mathbf{R}^{d}\right)}}{h^{|\alpha+\beta|} \alpha !^{s} \beta ! \sigma}\right),
$$

where the supremum is taken over all $\alpha_{j}, \beta_{j} \in \mathbf{N}^{d_{j}}, j=1, \ldots, n$. Then $f \mapsto\|f\|_{\mathcal{S}_{s ; h}^{\sigma}}$ 
defines a norm on $C^{\infty}\left(\mathbf{R}^{d}\right)$ which might attend infinity. The space $\mathcal{S}_{s ; h}^{\sigma}\left(\mathbf{R}^{d}\right)$ is the Banach space which consist of all $f \in C^{\infty}\left(\mathbf{R}^{d}\right)$ such that $\|f\|_{\mathcal{S}_{s: h}^{\sigma}}$ is finite. In the case $d_{1}=d \geq 1, d_{2}=\cdots=d_{n}=0, s=s_{1}, \sigma=\sigma_{1}$ and $x_{1}=x,(2.1)$ is interpreted as

$$
\|f\|_{\mathcal{S}_{s ; h}^{\sigma}} \equiv \sup _{\alpha, \beta \in \mathbf{N}^{d}}\left(\frac{\left\|x^{\alpha} \partial_{x}^{\beta} f(x)\right\|_{L^{\infty}\left(\mathbf{R}^{d}\right)}}{h^{|\alpha+\beta|} \alpha !^{s} \beta !^{\sigma}}\right)
$$

The Gelfand-Shilov spaces $\mathcal{S}_{s}^{\sigma}\left(\mathbf{R}^{d}\right)$ and $\Sigma_{s}^{\sigma}\left(\mathbf{R}^{d}\right)$ are defined as the inductive and projective limits, respectively of $\mathcal{S}_{s ; h}^{\sigma}\left(\mathbf{R}^{d}\right)$. This implies that

$$
\begin{aligned}
\mathcal{S}_{s}^{\boldsymbol{\sigma}}\left(\mathbf{R}^{d}\right) & =\bigcup_{h>0} \mathcal{S}_{s ; h}^{\boldsymbol{\sigma}}\left(\mathbf{R}^{d}\right), \\
\Sigma_{s}^{\boldsymbol{\sigma}}\left(\mathbf{R}^{d}\right) & =\bigcap_{h>0} \mathcal{S}_{s ; h}^{\boldsymbol{\sigma}}\left(\mathbf{R}^{d}\right),
\end{aligned}
$$

and that the topology for $\mathcal{S}_{s}^{\sigma}\left(\mathbf{R}^{d}\right)$ is the strongest possible one such that the inclusion map from $\mathcal{S}_{s ; h}^{\sigma}\left(\mathbf{R}^{d}\right)$ to $\mathcal{S}_{s}^{\sigma}\left(\mathbf{R}^{d}\right)$ is continuous, for every choice of $h>0$. The space

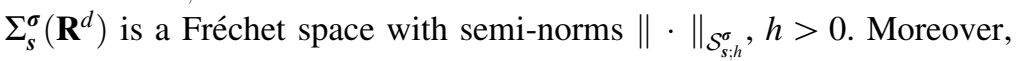

$$
\Sigma_{\boldsymbol{s}}^{\sigma}\left(\mathbf{R}^{d}\right) \neq\{0\} \quad \Leftrightarrow \quad s_{j}+\sigma_{j} \geq 1 \text { and }\left(s_{j}, \sigma_{j}\right) \neq\left(\frac{1}{2}, \frac{1}{2}\right), j=1, \ldots, n,
$$

and

$$
\mathcal{S}_{\boldsymbol{s}}^{\sigma}\left(\mathbf{R}^{d}\right) \neq\{0\} \quad \Leftrightarrow \quad s_{j}+\sigma_{j} \geq 1, j=1, \ldots, n .
$$

There are various kinds of characterizations of the spaces $\mathcal{S}_{\boldsymbol{s}}^{\boldsymbol{\sigma}}\left(\mathbf{R}^{d}\right)$ and $\Sigma_{\boldsymbol{s}}^{\boldsymbol{\sigma}}\left(\mathbf{R}^{d}\right)$, e. g., in terms of the exponential decay of their elements. Later on it will be useful that $f \in \mathcal{S}_{\boldsymbol{s}}^{\boldsymbol{\sigma}}\left(\mathbf{R}^{d}\right)$ (respectively, $f \in \Sigma_{\boldsymbol{s}}^{\boldsymbol{\sigma}}\left(\mathbf{R}^{d}\right)$ ), if and only if

$$
\left|\partial_{x}^{\alpha} f(x)\right| \lesssim h^{|\alpha|} \alpha !^{\sigma} e^{-r\left(\left|x_{1}\right|^{\frac{1}{s_{1}}}+\cdots+\left|x_{n}\right|^{\frac{1}{s_{n}}}\right)}
$$

for some $h, r>0$ (respectively, for every $h>0, r>0$ ).

If $\boldsymbol{I}=(1, \ldots, 1) \in \mathbf{R}^{n}$ and $\boldsymbol{s}, \boldsymbol{\sigma} \in \mathbf{R}_{+}^{n}$, then

$$
\Sigma_{\boldsymbol{s}}^{\boldsymbol{\sigma}}\left(\mathbf{R}^{d}\right) \hookrightarrow \mathcal{S}_{\boldsymbol{s}}^{\boldsymbol{\sigma}}\left(\mathbf{R}^{d}\right) \hookrightarrow \Sigma_{\boldsymbol{s}+\varepsilon \boldsymbol{I}}^{\boldsymbol{\sigma}+\varepsilon \boldsymbol{I}}\left(\mathbf{R}^{d}\right) \hookrightarrow \mathscr{S}\left(\mathbf{R}^{d}\right)
$$

for every $\varepsilon>0$. If in addition $s_{j}+\sigma_{j} \geq 1$ for every $j$, then the last two inclusions in (2.3) are dense, and if $s_{j}+\sigma_{j} \geq 1$ and $\left(s_{j}, \sigma_{j}\right) \neq\left(\frac{1}{2}, \frac{1}{2}\right)$ for every $j$, then the first inclusion in (2.3) is dense.

In order for discuss duality properties of Gelfand-Shilov spaces we first recall the definition of Gelfand tripples.

Definition 2.1 Let $\mathcal{V}, \mathcal{H}, \mathcal{V}^{\prime}$ be topological vector spaces. Then $\left(\mathcal{V}, \mathcal{H}, \mathcal{V}^{\prime}\right)$ is called a Gelfand tripple, if the following conditions are fulfilled:

(1) $\mathcal{V} \subseteq \mathcal{H} \subseteq \mathcal{V}^{\prime}$ 
(2) $\mathcal{H}$ is a Hilbert space and the restriction of the $\mathcal{H}$-scalar product $(\cdot, \cdot)_{\mathcal{H}}$ to $\mathcal{V} \times \mathcal{V}$ is uniquely extendable to continuous mappings from $\mathcal{V}^{\prime} \times \mathcal{V}$ to $\mathbf{C}$ and from $\mathcal{V} \times \mathcal{V}^{\prime}$ to $\mathbf{C}$;

(3) the dual space of $\mathcal{V}$ can be identified with $\mathcal{V}^{\prime}$ through the form $(\cdot, \cdot)_{\mathcal{H}}$ on $\mathcal{V}^{\prime} \times \mathcal{V}$ in (2).

The Gelfand-Shilov distribution spaces $\left(\mathcal{S}_{s}^{\sigma}\right)^{\prime}\left(\mathbf{R}^{d}\right)$ and $\left(\Sigma_{s}^{\sigma}\right)^{\prime}\left(\mathbf{R}^{d}\right)$ are the projective and inductive limits respectively of $\left(\mathcal{S}_{s ; h}^{\sigma}\right)^{\prime}\left(\mathbf{R}^{d}\right)$ with respect to $h>0$. Here $\left(\mathcal{S}_{s ; h}^{\sigma}\right)^{\prime}\left(\mathbf{R}^{d}\right)$ is the dual of $\mathcal{S}_{\boldsymbol{s} ; h}^{\sigma}\left(\mathbf{R}^{d}\right)$. This implies that

$$
\begin{aligned}
& \left(\mathcal{S}_{\boldsymbol{s}}^{\boldsymbol{\sigma}}\right)^{\prime}\left(\mathbf{R}^{d}\right)=\bigcap_{h>0}\left(\mathcal{S}_{\boldsymbol{s} ; h}^{\sigma}\right)^{\prime}\left(\mathbf{R}^{d}\right), \\
& \left(\Sigma_{\boldsymbol{s}}^{\boldsymbol{\sigma}}\right)^{\prime}\left(\mathbf{R}^{d}\right)=\bigcup_{h>0}\left(\mathcal{S}_{s ; h}^{\boldsymbol{\sigma}}\right)^{\prime}\left(\mathbf{R}^{d}\right) .
\end{aligned}
$$

If in addition $d_{1}=d \geq 1, d_{2}=\cdots=d_{n}=0, s=s_{1}$ and $\sigma_{1}=\sigma$, then we set $\left(\mathcal{S}_{s}^{\sigma}\right)^{\prime}\left(\mathbf{R}^{d}\right)=\left(\mathcal{S}_{s}^{\sigma}\right)^{\prime}\left(\mathbf{R}^{d}\right)$ and $\left(\Sigma_{s}^{\sigma}\right)^{\prime}\left(\mathbf{R}^{d}\right)=\left(\Sigma_{s}^{\sigma}\right)^{\prime}\left(\mathbf{R}^{d}\right)$. We remark that the analysis in [12] shows that

$$
\left(\mathcal{S}_{\boldsymbol{s}}^{\boldsymbol{\sigma}}\left(\mathbf{R}^{d}\right), L^{2}\left(\mathbf{R}^{d}\right),\left(\mathcal{S}_{\boldsymbol{s}}^{\boldsymbol{\sigma}}\right)^{\prime}\left(\mathbf{R}^{d}\right)\right)
$$

is a Gelfand tripple when $s_{j}+\sigma_{j} \geq 1, j=1, \ldots, n$, and that

$$
\left(\Sigma_{\boldsymbol{s}}^{\boldsymbol{\sigma}}\left(\mathbf{R}^{d}\right), L^{2}\left(\mathbf{R}^{d}\right),\left(\Sigma_{\boldsymbol{s}}^{\boldsymbol{\sigma}}\right)^{\prime}\left(\mathbf{R}^{d}\right)\right)
$$

is a Gelfand tripple when $s_{j}+\sigma_{j} \geq 1$ and $\left(s_{j}, \sigma_{j}\right) \neq\left(\frac{1}{2}, \frac{1}{2}\right), j=1, \ldots, n$. In particular, $\left(\mathcal{S}_{\boldsymbol{s}}^{\sigma}\right)^{\prime}\left(\mathbf{R}^{d}\right)$ and $\left(\Sigma_{\boldsymbol{s}}^{\sigma}\right)^{\prime}\left(\mathbf{R}^{d}\right)$ are the topological duals of $\mathcal{S}_{\boldsymbol{s}}^{\sigma}\left(\mathbf{R}^{d}\right)$ respectively, $\Sigma_{\boldsymbol{s}}^{\boldsymbol{\sigma}}\left(\mathbf{R}^{d}\right)$ through unique extensions of the $L^{2}$-scalar product on $\mathcal{S}_{s}^{\sigma}\left(\mathbf{R}^{d}\right) \times \mathcal{S}_{s}^{\sigma}\left(\mathbf{R}^{d}\right)$ respectively, on $\Sigma_{\boldsymbol{s}}^{\boldsymbol{\sigma}}\left(\mathbf{R}^{d}\right) \times \Sigma_{\boldsymbol{s}}^{\boldsymbol{\sigma}}\left(\mathbf{R}^{d}\right)$ to continuous mappings from $\left(\mathcal{S}_{\boldsymbol{s}}^{\sigma}\right)^{\prime}\left(\mathbf{R}^{d}\right) \times \mathcal{S}_{\boldsymbol{s}}^{\boldsymbol{\sigma}}\left(\mathbf{R}^{d}\right)$ respectively, $\left(\Sigma_{s}^{\sigma}\right)^{\prime}\left(\mathbf{R}^{d}\right) \times \Sigma_{s}^{\sigma}\left(\mathbf{R}^{d}\right)$ to $\mathbf{C}$.

By the inequalities $n ! k ! \leq(n+k) ! \leq 2^{n+k} n ! k !$ it follows that

$$
\begin{aligned}
\mathcal{S}_{s, \ldots, s}^{\sigma, \ldots, \sigma}\left(\mathbf{R}^{d}\right) & =\mathcal{S}_{s}^{\sigma}\left(\mathbf{R}^{d}\right), & \Sigma_{s, \ldots, s}^{\sigma, \ldots, \sigma}\left(\mathbf{R}^{d}\right) & =\Sigma_{s}^{\sigma}\left(\mathbf{R}^{d}\right), \\
\left(\mathcal{S}_{s, \ldots, s}^{\sigma, \ldots, \sigma}\right)^{\prime}\left(\mathbf{R}^{d}\right) & =\left(\mathcal{S}_{s}^{\sigma}\right)^{\prime}\left(\mathbf{R}^{d}\right), & \left(\Sigma_{s, \ldots, s}^{\sigma, \ldots, \sigma}\right)^{\prime}\left(\mathbf{R}^{d}\right) & =\left(\Sigma_{s}^{\sigma}\right)^{\prime}\left(\mathbf{R}^{d}\right),
\end{aligned}
$$

Corresponding relations to (2.3) for Gelfand-Shilov distributions are

$$
\mathscr{S}^{\prime}\left(\mathbf{R}^{d}\right) \hookrightarrow\left(\Sigma_{\boldsymbol{s}+\varepsilon \boldsymbol{I}}^{\boldsymbol{\sigma}+\varepsilon \boldsymbol{I}}\right)^{\prime}\left(\mathbf{R}^{d}\right) \hookrightarrow\left(\mathcal{S}_{\boldsymbol{s}}^{\boldsymbol{\sigma}}\right)^{\prime}\left(\mathbf{R}^{d}\right)
$$

when $s_{j}+\sigma_{j} \geq 1, j=1, \ldots, n$, and

$$
\left(\mathcal{S}_{\boldsymbol{s}}^{\boldsymbol{\sigma}}\right)^{\prime}\left(\mathbf{R}^{d}\right) \hookrightarrow\left(\Sigma_{\boldsymbol{s}}^{\boldsymbol{\sigma}}\right)^{\prime}\left(\mathbf{R}^{d}\right)
$$

when $s_{j}+\sigma_{j} \geq 1$ and $\left(s_{j}, \sigma_{j}\right) \neq\left(\frac{1}{2}, \frac{1}{2}\right), j=1, \ldots, n$.

The Gelfand-Shilov spaces and their distribution spaces possess several convenient properties. For example they are complete, invariant under translations, 
dilations, and to some extent (partial) Fourier transformations. For any $f \in L^{1}\left(\mathbf{R}^{d}\right)$, its Fourier transform is defined by

$$
(\mathscr{F} f)(\xi)=\widehat{f}(\xi) \equiv(2 \pi)^{-\frac{d}{2}} \int_{\mathbf{R}^{d}} f(x) e^{-i\langle x, \xi\rangle} d x .
$$

If instead $f \in L^{1}\left(\mathbf{R}^{d_{1}+\cdots+d_{n}}\right)$, then the partial Fourier transform of $f$ with respect to $k \in\{1, \ldots, n\}$ is given by

$$
\begin{aligned}
& \left(\mathscr{F}_{k} f\right)\left(x_{1}, \ldots, \xi_{k}, \ldots, x_{n}\right) \\
& \quad \equiv(2 \pi)^{-\frac{d_{k}}{2}} \int_{\mathbf{R}^{d_{k}}} f\left(x_{1}, \ldots, x_{k}, \ldots, x_{n}\right) e^{-i\left\langle x_{k}, \xi_{k}\right\rangle} d x_{k}, \quad x_{j}, \xi_{j} \in \mathbf{R}^{d_{j}} .
\end{aligned}
$$

Remark 2.2 Let $d=d_{1}+\cdots+d_{n}, j \in\{1, \ldots, n\}, \boldsymbol{s}, \boldsymbol{\sigma} \in \mathbf{R}_{+}^{n}, \tau_{k, j}(\boldsymbol{s}, \boldsymbol{\sigma})=\left(r_{k, j}, \ldots\right.$ ,$\left.r_{k, j, n}\right), k=1,2$, where

$$
r_{1, j, l}=\left\{\begin{array}{ll}
s_{l}, & l \neq j \\
\sigma_{l}, & l=j,
\end{array} \text { and } \quad r_{2, j, l}= \begin{cases}\sigma_{l}, & l \neq j \\
s_{l}, & l=j .\end{cases}\right.
$$

Then the following assertions follow from the general theory of Schwartz functions and Gelfand-Shilov functions and their distributions (see, e.g. [5, 9]):

(1) the definition of $\mathscr{F}_{j}$ extends to a homeomorphism on $\mathscr{S}^{\prime}\left(\mathbf{R}^{d}\right)$ and restricts to a homeomorphism on $\mathscr{S}\left(\mathbf{R}^{d}\right)$;

(2) the definition of $\mathscr{F}_{j}$ extends uniquely to a homeomorphism from $\left(\mathcal{S}_{s}^{\sigma}\right)^{\prime}\left(\mathbf{R}^{d}\right)$ to $\left(\mathcal{S}_{\tau_{1, j}(\boldsymbol{s}, \boldsymbol{\sigma})}^{\tau_{2, j}(\boldsymbol{s}, \boldsymbol{\sigma})}\right)^{\prime}\left(\mathbf{R}^{d}\right)$, and from $\left(\Sigma_{\boldsymbol{s}}^{\boldsymbol{\sigma}}\right)^{\prime}\left(\mathbf{R}^{d}\right)$ to $\left(\Sigma_{\tau_{1, j}(\boldsymbol{s}, \boldsymbol{\sigma})}^{\tau_{2, j}(\boldsymbol{s}, \boldsymbol{\sigma})}\right)^{\prime}\left(\mathbf{R}^{d}\right)$;

(3) $\mathscr{F}_{j}$ restricts to homeomorphisms from $\mathcal{S}_{\boldsymbol{s}}^{\sigma}\left(\mathbf{R}^{d}\right)$ to $\mathcal{S}_{\tau_{1, j}(\boldsymbol{s}, \boldsymbol{\sigma})}^{\tau_{2, j}(\boldsymbol{s}, \boldsymbol{\sigma})}\left(\mathbf{R}^{d}\right)$, and from $\Sigma_{\boldsymbol{s}}^{\boldsymbol{\sigma}}\left(\mathbf{R}^{d}\right)$ to $\Sigma_{\tau_{1, j}(\boldsymbol{s}, \boldsymbol{\sigma})}^{\tau_{2, j}(\boldsymbol{s}, \boldsymbol{\sigma}}\left(\mathbf{R}^{d}\right)$.

Remark 2.3 There are several characterizations of Gelfand-Shilov spaces in the literature. For example, let $s, \sigma \in \mathbf{R}_{+}$and $p \in[1, \infty]$. Then it is proved in [5] that $\psi \in \mathscr{S}^{\prime}\left(\mathbf{R}^{d}\right)$ belongs to $\mathcal{S}_{s}^{\sigma}\left(\mathbf{R}^{d}\right)\left(\Sigma_{s}^{\sigma}\left(\mathbf{R}^{d}\right)\right)$, if and only if

$$
\|\psi\|_{(r, p)} \equiv\left\|\psi \cdot e^{r|\cdot|^{\frac{1}{s}}}\right\|_{L^{p}}+\left\|\widehat{\psi} \cdot e^{r|\cdot|^{\frac{1}{\sigma}}}\right\|_{L^{p}}
$$

is finite for some $r>0$ (for every $r>0$ ). Furthermore, the topology of $\mathcal{S}_{s}^{\sigma}\left(\mathbf{R}^{d}\right.$ ) $\left(\Sigma_{s}^{\sigma}\left(\mathbf{R}^{d}\right)\right)$ is obtained by the inductive (projective) limit topologies, supplied by the semi-norms $\|\cdot\|_{(r, p)}$ with respect to $r>0$.

\subsection{Pilipović spaces}

Next we make a review of Pilipović spaces. These spaces can be defined in terms of Hermite series expansions. We recall that the Hermite function of order $\alpha \in \mathbf{N}^{d}$ is defined by 


$$
h_{\alpha}(x)=\pi^{-\frac{d}{4}}(-1)^{|\alpha|}\left(2^{|\alpha|} \alpha !\right)^{-\frac{1}{2}} e^{\frac{1}{2} \cdot|x|^{2}}\left(\partial^{\alpha} e^{-|x|^{2}}\right) .
$$

It follows that

$$
h_{\alpha}(x)=\left((2 \pi)^{\frac{d}{2}} \alpha !\right)^{-1} p_{\alpha}(x) e^{\frac{1}{2 \cdot} \cdot|x|^{2}},
$$

for some polynomial $p_{\alpha}$ on $\mathbf{R}^{d}$, which is called the Hermite polynomial of order $\alpha$. The Hermite functions are eigenfunctions to the Fourier transform, partial Fourier transforms and to the Harmonic oscillator $H_{d} \equiv|x|^{2}-\Delta$ which acts on functions and (ultra-)distributions defined on $\mathbf{R}^{d}$. For example, we have

$$
H_{d} h_{\alpha}=(2|\alpha|+d) h_{\alpha}, \quad H_{d} \equiv|x|^{2}-\Delta .
$$

It is well-known that the set of Hermite functions is a basis for $\mathscr{S}\left(\mathbf{R}^{d}\right)$ and an orthonormal basis for $L^{2}\left(\mathbf{R}^{d}\right)$ (cf. [14]). In particular, if $f \in L^{2}\left(\mathbf{R}^{d}\right)$, then

$$
\|f\|_{L^{2}\left(\mathbf{R}^{d}\right)}^{2}=\sum_{\alpha \in \mathbf{N}^{d}}\left|c_{h}(f, \alpha)\right|^{2},
$$

where

$$
f(x)=\sum_{\alpha \in \mathbf{N}^{d}} c_{h}(f, \alpha) h_{\alpha}
$$

is the Hermite seriers expansion of $f$, and

$$
c_{h}(f, \alpha)=\left(f, h_{\alpha}\right)_{L^{2}\left(\mathbf{R}^{d}\right)}
$$

is the Hermite coefficient of $f$ of order $\alpha \in \mathbf{R}^{d}$.

In order to define the full scale of Pilipović spaces, their order $s$ should belong to the extended set

$$
\mathbf{R}_{b}=\mathbf{R}_{+} \bigcup\left\{b_{\sigma} ; \sigma \in \mathbf{R}_{+}\right\},
$$

of $\mathbf{R}_{+}$, with extended inequality relations as

$$
s_{1}<b_{\sigma}<s_{2} \text { and } b_{\sigma_{1}}<b_{\sigma_{2}}
$$

when $s_{1}<\frac{1}{2} \leq s_{2}$ and $\sigma_{1}<\sigma_{2}$. (Cf. [16].)

For $r>0$ and $s \in \mathbf{R}_{b}$ we set

$$
\vartheta_{r, s}(\alpha) \equiv\left\{\begin{array}{cc}
e^{-r|\alpha|^{\frac{1}{2 s}}}, & s \in \mathbf{R}_{+}, \\
r^{|\alpha|} \alpha !^{-\frac{1}{2 \sigma}}, & s=b_{\sigma},
\end{array}\right.
$$

and

$$
\vartheta_{r, s}^{\prime}(\alpha) \equiv\left\{\begin{array}{cc}
e^{r|\alpha|^{\frac{1}{2 s}}}, & s \in \mathbf{R}_{+}, \\
r^{|\alpha|} \alpha !^{\frac{1}{2 \sigma}}, & s=b_{\sigma} .
\end{array}\right.
$$


Definition 2.4 Let $s \in \overline{\mathbf{R}_{b}}=\mathbf{R}_{b} \cup\{0\}$, and let $\vartheta_{r, s}$ and $\vartheta_{r, s}^{\prime}$ be as in (2.7) and (2.8).

(1) $\mathcal{H}_{0}\left(\mathbf{R}^{d}\right)$ consists of all finite expansions in (2.5), and $\mathcal{H}_{0}^{\prime}\left(\mathbf{R}^{d}\right)$ consists of all formal Hermite series expansions in (2.5);

(2) if $s \in \mathbf{R}_{b}$, then $\mathcal{H}_{s}\left(\mathbf{R}^{d}\right)\left(\mathcal{H}_{0, s}\left(\mathbf{R}^{d}\right)\right)$ consists of all $f \in L^{2}\left(\mathbf{R}^{d}\right)$ such that

$$
\left|c_{h}\left(f, h_{\alpha}\right)\right| \lesssim \vartheta_{r, s}(\alpha)
$$

holds true for some $r \in \mathbf{R}_{+}$(for every $r \in \mathbf{R}_{+}$);

(3) if $s \in \mathbf{R}_{b}$, then $\mathcal{H}_{s}^{\prime}\left(\mathbf{R}^{d}\right)\left(\mathcal{H}_{0, s}^{\prime}\left(\mathbf{R}^{d}\right)\right)$ consists of all formal Hermite series expansions in (2.5) such that

$$
\left|c_{h}\left(f, h_{\alpha}\right)\right| \lesssim \vartheta_{r, s}^{\prime}(\alpha)
$$

holds true for every $r \in \mathbf{R}_{+}$(for some $r \in \mathbf{R}_{+}$).

The spaces $\mathcal{H}_{s}\left(\mathbf{R}^{d}\right)$ and $\mathcal{H}_{0, s}\left(\mathbf{R}^{d}\right)$ are called Pilipovic spaces of Roumieu respectively Beurling types of order $s$, and $\mathcal{H}_{s}^{\prime}\left(\mathbf{R}^{d}\right)$ and $\mathcal{H}_{0, s}^{\prime}\left(\mathbf{R}^{d}\right)$ are called Pilipović distribution spaces of Roumieu respectively Beurling types of order $s$.

Remark 2.5 Let $\mathcal{S}_{s}\left(\mathbf{R}^{d}\right) \equiv \mathcal{S}_{s}^{s}\left(\mathbf{R}^{d}\right)$ and $\Sigma_{s}\left(\mathbf{R}^{d}\right) \equiv \Sigma_{s}^{s}\left(\mathbf{R}^{d}\right)$ be the Fourier invariant Gelfand-Shilov spaces of order $s \in \mathbf{R}_{+}$and of Roumieu and Beurling types, respectively. Then it is proved in $[11,12]$ that

$$
\begin{array}{cc}
\mathcal{H}_{0, s}\left(\mathbf{R}^{d}\right)=\Sigma_{s}\left(\mathbf{R}^{d}\right) \neq\{0\}, & s>\frac{1}{2} \\
\mathcal{H}_{0, s}\left(\mathbf{R}^{d}\right) \neq \Sigma_{s}\left(\mathbf{R}^{d}\right)=\{0\}, & s \leq \frac{1}{2} \\
\mathcal{H}_{s}\left(\mathbf{R}^{d}\right)=\mathcal{S}_{s}\left(\mathbf{R}^{d}\right) \neq\{0\}, & s \geq \frac{1}{2}
\end{array}
$$

and

$$
\mathcal{H}_{s}\left(\mathbf{R}^{d}\right) \neq \mathcal{S}_{s}\left(\mathbf{R}^{d}\right)=\{0\}, \quad s<\frac{1}{2}
$$

Next we recall the topologies for Pilipović spaces. Let $s \in \mathbf{R}_{b}, r>0$, and let $\|f\|_{\mathcal{H}_{s ; r}}$ and $\|f\|_{\mathcal{H}_{s ; r}^{\prime}}$ be given by

$$
\|f\|_{\mathcal{H}_{s ; r}} \equiv \sup _{\alpha \in \mathbf{N}^{d}}\left|c_{h}(f, \alpha) / \vartheta_{r, s}(\alpha)\right|, \quad s \in \mathbf{R}_{b},
$$

and

$$
\|f\|_{\mathcal{H}_{s, r}^{\prime}} \equiv \sup _{\alpha \in \mathbf{N}^{d}}\left|c_{h}(f, \alpha) / \vartheta_{r, s}^{\prime}(\alpha)\right|, \quad s \in \mathbf{R}_{b}
$$

when $f$ is a formal expansion in (2.5). Then $\mathcal{H}_{s ; r}\left(\mathbf{R}^{d}\right)$ consists of all expansions (2.5) such that $\|f\|_{\mathcal{H}_{s ; r}}$ is finite, and $\mathcal{H}_{s ; r}^{\prime}\left(\mathbf{R}^{d}\right)$ consists of all expansions (2.5) such that 
$\|f\|_{\mathcal{H}_{s, r}^{\prime}}$ is finite. It follows that both $\mathcal{H}_{s ; r}\left(\mathbf{R}^{d}\right)$ and $\mathcal{H}_{s ; r}^{\prime}\left(\mathbf{R}^{d}\right)$ are Banach spaces under the norms $f \longmapsto\|f\|_{\mathcal{H}_{s ; r}}$ and $f \mapsto\|f\|_{\mathcal{H}_{s: r}^{\prime}}$, respectively.

We let the topologies of $\mathcal{H}_{s}\left(\mathbf{R}^{d}\right)$ and $\mathcal{H}_{0, s}\left(\mathbf{R}^{d}\right)$ be the inductive respectively, projective limit topology of $\mathcal{H}_{s ; r}\left(\mathbf{R}^{d}\right)$ with respect to $r>0$. In the same way, the topologies of $\mathcal{H}_{s}^{\prime}\left(\mathbf{R}^{d}\right)$ and $\mathcal{H}_{0, s}^{\prime}\left(\mathbf{R}^{d}\right)$ are the projective respectively, inductive limit topology of $\mathcal{H}_{s ; r}^{\prime}\left(\mathbf{R}^{d}\right)$ with respect to $r>0$.

Suppose instead $s=0$. For any integer $N \geq 0$, we set

$$
\|f\|_{(N)} \equiv \sup _{|\alpha| \leq N}\left|c_{h}(f, \alpha)\right|, \quad f \in \mathcal{H}_{0}^{\prime}\left(\mathbf{R}^{d}\right)
$$

The topology for $\mathcal{H}_{0}^{\prime}\left(\mathbf{R}^{d}\right)$ is defined by the semi-norms $\|\cdot\|_{(N)}$.

We also let $\mathcal{H}_{0}^{(N)}\left(\mathbf{R}^{d}\right)$ be the vector space which consists of all $f \in \mathcal{H}_{0}^{\prime}\left(\mathbf{R}^{d}\right)$ such that $c_{h}(f, \alpha)=0$ when $|\alpha|>N$, and equip this space with the topology, defined by the norm $\|\cdot\|_{(N)}$. The topology of $\mathcal{H}_{0}\left(\mathbf{R}^{d}\right)$ is then defined as the inductive limit topology of $\mathcal{H}_{0}^{(N)}\left(\mathbf{R}^{d}\right)$ with respect to $N \geq 0$.

It follows that the spaces in Definition 2.4 are complete, and that $\mathcal{H}_{0, s}\left(\mathbf{R}^{d}\right)$ and $\mathcal{H}_{s}^{\prime}\left(\mathbf{R}^{d}\right)$ are Fréchet space with semi-norms $f \mapsto\|f\|_{\mathcal{H}_{s ; r}}$ and $f \mapsto\|f\|_{\mathcal{H}_{s ; r}^{\prime}}$, respectively. By [16] it follows that

$$
\left(\mathcal{H}_{s}\left(\mathbf{R}^{d}\right), L^{2}\left(\mathbf{R}^{d}\right), \mathcal{H}_{s}^{\prime}\left(\mathbf{R}^{d}\right)\right)
$$

is a Gelfand tripple when $s \in \overline{\mathbf{R}}_{b}$ and that

$$
\left(\mathcal{H}_{0, s}\left(\mathbf{R}^{d}\right), L^{2}\left(\mathbf{R}^{d}\right), \mathcal{H}_{0, s}^{\prime}\left(\mathbf{R}^{d}\right)\right)
$$

is a Gelfand tripple when $s \in \mathbf{R}_{b}$. Obviously, if $\langle\cdot, \cdot\rangle$ denotes the action between (ultra-)distributions and their corresponding (ultra-)test functions, then $\langle f, \varphi\rangle=(f, \bar{\varphi})=(f, \bar{\varphi})_{L^{2}}$, when $f \in \mathcal{H}_{s}^{\prime}\left(\mathbf{R}^{d}\right)$ and $\varphi \in \mathcal{H}_{s}\left(\mathbf{R}^{d}\right)$. The same holds true with $\mathcal{H}_{0, s}, \mathcal{S}_{s}^{\sigma}$ or $\Sigma_{s}^{\sigma}$ in place of $\mathcal{H}_{s}$ at each occurrence.

The following characterizations of Pilipović spaces can be found in [16]. The proof is therefore omitted.

Proposition 2.6 Let $s \in \mathbf{R}_{+} \cup\{0\}$ and let $f \in \mathcal{H}_{0}^{\prime}\left(\mathbf{R}^{d}\right)$. Then $f \in \mathcal{H}_{0, s}\left(\mathbf{R}^{d}\right)$ $\left(f \in \mathcal{H}_{s}\left(\mathbf{R}^{d}\right)\right)$, if and only if $f \in C^{\infty}\left(\mathbf{R}^{d}\right)$ and satisfies $\left|H_{d}^{N} f(x)\right| \lesssim h^{N} N !^{2 s}$ for every $h>0$ (for some $h>0$ ).

Finally we remark that the Pilipović spaces of functions and distributions possess convenient mapping properties under the Bargmann transform (cf. [16] and Remarks 4.4 and 4.5 in Sect. 4).

\section{Tensor product for Gelfand-Shilov spaces}

In this section we start by proving Theorem 1.2. Thereafter we deduce a multi-linear version of this result. 
For the proof of Theorem 1.2 we first need the following analogy of Lemma 4.1.3 in [9].

Lemma 3.1 Let $s_{1}, s_{2}, \sigma_{1}, \sigma_{2}>0, \varphi, \psi \in \mathcal{S}_{s_{1}, s_{2}}^{\sigma_{1}, \sigma_{2}}\left(\mathbf{R}^{d_{1}+d_{2}}\right)$. Then the Riemann sum

$$
\sum_{k \in \mathbf{Z}^{d}} \varphi(x-\varepsilon k) \psi(\varepsilon k) \varepsilon^{d}, \quad d=d_{1}+d_{2},
$$

converges to $(\varphi * \psi)(x)$ in $\mathcal{S}_{s_{1}, s_{2}}^{\sigma_{1}, \sigma_{2}}\left(\mathbf{R}^{d_{1}+d_{2}}\right)$ as $\varepsilon \rightarrow 0$.

The same holds true if each $\mathcal{S}_{S_{1}, S_{2}}^{\sigma_{1}, \sigma_{2}}$ are replaced by $\Sigma_{s_{1}, S_{2}}^{\sigma_{1}, \sigma_{2}}$.

Proof We may assume that $\varepsilon>0$, and consider first the case when $\varphi$ and $\psi$ are real-valued. Set

$$
R_{\varepsilon, \alpha, \beta}(x)=x^{\alpha} D_{x}^{\beta}\left(\int_{\mathbf{R}^{d}} \varphi(x-y) \psi(y) d y-\sum_{k \in \mathbf{Z}^{d}} \varphi(x-\varepsilon k) \psi(\varepsilon k) \varepsilon^{d}\right) .
$$

and $Q_{d, r}=[0, r]^{d}$. By the mean-value theorem we have for some $\rho_{k}=\rho_{k}(x, y) \in Q_{d, 1}, k \in \mathbf{Z}^{d}$ that

$$
\begin{aligned}
\left|R_{\varepsilon, \alpha, \beta}(x)\right|= & \left|\int_{\mathbf{R}^{d}} x^{\alpha}\left(D_{x}^{\beta} \varphi\right)(x-y) \psi(y) d y-\sum_{k \in \mathbf{Z}^{d}} x^{\alpha}\left(D_{x}^{\beta} \varphi\right)(x-\varepsilon k) \psi(\varepsilon k) \varepsilon^{d}\right| \\
& =\left|\sum_{k \in \mathbf{Z}^{d}}\left(\int_{\varepsilon k+Q_{d, s}} x^{\alpha}\left(D_{x}^{\beta} \varphi\right)(x-y) \psi(y) d y-x^{\alpha}\left(D_{x}^{\beta} \varphi\right)(x-\varepsilon k) \psi(\varepsilon k) \varepsilon^{d}\right)\right| \\
& =\left|\sum_{k \in \mathbf{Z}^{d}}\left(x^{\alpha}\left(D_{x}^{\beta} \varphi\right)\left(x-\varepsilon k-\varepsilon \rho_{k}\right) \psi\left(\varepsilon k+\varepsilon \rho_{k}\right)-x^{\alpha}\left(D_{x}^{\beta} \varphi\right)(x-\varepsilon k) \psi(\varepsilon k)\right) \varepsilon^{d}\right| \\
& \leq \sum_{k \in \mathbf{Z}^{d}}\left|x^{\alpha}\left(D_{x}^{\beta} \varphi\right)\left(x-\varepsilon k-\varepsilon \rho_{k}\right) \psi\left(\varepsilon k+\varepsilon \rho_{k}\right)-x^{\alpha}\left(D_{x}^{\beta} \varphi\right)(x-\varepsilon k) \psi(\varepsilon k)\right| \varepsilon^{d} \\
& \leq \sum_{k \in \mathbf{Z}^{d}} \sum_{j=1}^{d} \sup _{z \in Q_{d, s}}\left|D_{z_{j}}\left(x^{\alpha}\left(D_{x}^{\beta} \varphi\right)(x-\varepsilon k-z) \psi(\varepsilon k+z)\right)\right| \varepsilon^{d+1} \leq J_{1}+J_{2},
\end{aligned}
$$

where

$$
J_{1}=\sum_{\gamma \leq \alpha} \sum_{j=1}^{d} \sum_{k \in \mathbf{Z}^{d}}\left(\begin{array}{l}
\alpha \\
\gamma
\end{array}\right) \sup _{y \in \varepsilon k+Q_{d, \varepsilon}}\left|(x-y)^{\gamma} D_{x_{j}} D_{x}^{\beta} \varphi(x-y) y^{\alpha-\gamma} \psi(y)\right| \varepsilon^{d+1}
$$

and

$$
J_{2}=\sum_{\gamma \leq \alpha} \sum_{j=1}^{d} \sum_{k \in \mathbf{Z}^{d}}\left(\begin{array}{l}
\alpha \\
\gamma
\end{array}\right) \sup _{y \in \varepsilon k+Q_{d, \varepsilon}}\left|(x-y)^{\gamma} D_{x}^{\beta} \varphi(x-y) y^{\alpha-\gamma} D_{y_{j}} \psi(y)\right| \varepsilon^{d+1} .
$$

Since $(m+1) ! \leq 2^{m} m$ ! when $m \geq 0$ is an integer, and $\varphi, \psi \in \mathcal{S}_{S_{1}, S_{2}}^{\sigma_{1}, S_{2}}\left(\mathbf{R}^{d_{1}+d_{2}}\right)$, we get 


$$
\begin{aligned}
J_{j} & \lesssim h^{|\alpha+\beta|} 2^{\sigma|\beta|} d \sum_{\gamma \leq \alpha} \sum_{k \in \mathbf{Z}^{d}}\left(\begin{array}{c}
\alpha \\
\gamma
\end{array}\right) \gamma !^{s} \beta !^{\sigma} \sup _{y \in \varepsilon k+Q_{d, \varepsilon}} \mid y^{\alpha-\gamma} e^{-2 r\left(\left|y_{1}\right|^{\frac{1}{s_{1}}}+\left|y_{2}\right|^{\frac{1}{s_{2}}}\right) \mid \varepsilon^{d+1}} \\
& \lesssim \varepsilon h^{|\alpha+\beta|} 2^{\sigma|\beta|} \sum_{\gamma \leq \alpha} \sum_{k \in \mathbf{Z}^{d}}\left(\begin{array}{l}
\alpha \\
\gamma
\end{array}\right) \gamma !^{s} \beta !^{\sigma}(\alpha-\gamma) !^{s} e^{-r\left(\left|\varepsilon k_{1}\right|^{\frac{1}{s_{1}}}+\left|\varepsilon k_{2}\right|^{\frac{1}{s_{2}}}\right)} \varepsilon^{d} \\
& \lesssim \varepsilon\left(2^{\sigma} 2 h\right)^{|\alpha+\beta|} \alpha !^{s} \beta !^{\sigma} \sum_{k \in \mathbf{Z}^{d}} e^{-r\left(\left|\varepsilon k_{1}\right|^{\frac{1}{s_{1}}}+\left|\varepsilon k_{2}\right|^{\frac{1}{s_{2}}}\right)} \varepsilon^{d} \\
& \lesssim \varepsilon\left(2^{\sigma} 2 h\right)^{|\alpha+\beta|} \alpha !^{s} \beta !^{\sigma} \iint_{\mathbf{R}^{d}} e^{-r\left(\left|x_{1}\right|^{\frac{1}{s_{1}}}+\left|x_{2}\right|^{\frac{1}{s_{2}}}\right)} d x_{1} d x_{2} \asymp \varepsilon\left(2^{\sigma} 2 h\right)^{|\alpha+\beta|} \alpha !^{s} \beta !^{\sigma},
\end{aligned}
$$

$j=1,2$, for some positive constants $h$ and $r$. This implies that for some $h>0$ we have

$$
\sup _{\alpha, \beta \in \mathbf{N}^{d}}\left(\frac{\left\|R_{\varepsilon, \alpha, \beta}\right\|_{L^{\infty}}}{h^{|\alpha+\beta| \alpha !^{s} \beta !^{\sigma}}}\right) \leq C \varepsilon
$$

for some positive constants $C$ and $h$ which are independent of $\varepsilon$.

Since the right-hand side tends to zero when $\varepsilon>0$ tends to zero, the stated convergence follows in this case.

The general case follows from the previous case, after writing $\varphi=\varphi_{1}+i \varphi_{2}$ and $\psi=\psi_{1}+i \psi_{2}$ with $\varphi_{j}$ and $\psi_{j}$ being real-valued, $j=1,2$, giving that $\varphi * \psi$ is a superposition of $\varphi_{j_{1}} * \psi_{j_{2}}, j_{1}, j_{2} \in\{1,2\}$, and using the fact that $\varphi_{j} \in \mathcal{S}_{S_{1}, s_{2}}^{\sigma_{1}, \sigma_{2}}\left(\mathbf{R}^{d_{1}+d_{2}}\right)$ when $\varphi \in \mathcal{S}_{S_{1}, s_{2}}^{\sigma_{1}, \sigma_{2}}\left(\mathbf{R}^{d_{1}+d_{2}}\right)$.

We may now prove the following result related to [9, Theorem 4.1.2]

Lemma 3.2 Let $s_{1}, s_{2}, \sigma_{1}, \sigma_{2}>0, \quad \varphi, \psi \in \mathcal{S}_{S_{1}, s_{2}}^{\sigma_{1}, \sigma_{2}}\left(\mathbf{R}^{d_{1}+d_{2}}\right)$ and let $f \in\left(\mathcal{S}_{s_{1}, s_{2}}^{\sigma_{1}, \sigma_{2}}\right)^{\prime}$ $\left(\mathbf{R}^{d_{1}+d_{2}}\right)$. Then

$$
(f * \varphi) * \psi=f *(\varphi * \psi) .
$$

The same holds true if each $\mathcal{S}_{S_{1}, S_{2}}^{\sigma_{1}, \sigma_{2}}$ and $\left(\mathcal{S}_{S_{1}, S_{2}}^{\sigma_{1}, \sigma_{2}}\right)^{\prime}$ are replaced by $\Sigma_{s_{1}, S_{2}}^{\sigma_{1}, \sigma_{2}}$ and $\left(\Sigma_{s_{1}, S_{2}}^{\sigma_{1}, \sigma_{2}}\right)^{\prime}$, respectively.

Proof We use the same notations as in the previous proof. Since the Riemann sum in Lemma 3.1 converges to $\varphi * \psi$ in $\mathcal{S}_{S_{1}, s_{2}}^{\sigma_{1}, \sigma_{2}}$, we get

$$
\begin{aligned}
(f *(\varphi * \psi))(x) & =\lim _{\varepsilon \rightarrow 0}\left\langle f, \sum_{k \in \mathbf{Z}^{d}} \varphi(x-\cdot-\varepsilon k) \psi(\varepsilon k) \varepsilon^{d}\right\rangle \\
& =\lim _{\varepsilon \rightarrow 0}\left(\sum_{k \in \mathbf{Z}^{d}}(f * \varphi)(x-\varepsilon k) \psi(\varepsilon k) \varepsilon^{d}\right) .
\end{aligned}
$$

Here the second equality follows by the fact that

$$
y \longmapsto \sum_{k \in \mathbf{Z}^{d}} \varphi(x-y-\varepsilon k) \psi(\varepsilon k)
$$


converges in $\mathcal{S}_{S_{1}, s_{2}}^{\sigma_{1}, \sigma_{2}}$ for every fixed $x \in \mathbf{R}^{d}$ and $\varepsilon \in \mathbf{R}$.

We have that $f * \varphi$ is smooth, and for some $r_{0}>0$ we have

$$
|(f * \varphi)(x-\varepsilon k) \psi(\varepsilon k)| \lesssim e^{r\left(\left|x_{1}-\varepsilon k_{1}\right|^{\frac{1}{s_{1}}}+\left|x_{2}-\varepsilon k_{2}\right|^{\frac{1}{s_{2}}}\right)} e^{-2 r_{0}\left(\left|\varepsilon k_{1}\right|^{\frac{1}{s_{1}}}+\left|\varepsilon k_{2}\right|^{\frac{1}{s_{2}}}\right)}
$$

for every $r>0$. This gives

$$
|(f * \varphi)(x-\varepsilon k) \psi(\varepsilon k)| \leq C_{x} e^{-r_{0}\left(\left|\varepsilon k_{1}\right|^{\frac{1}{s_{1}}}+\left|\varepsilon k_{2}\right|^{\frac{1}{s_{2}}}\right)},
$$

for some constant $C_{x}$ which only depends on $x$ and $r_{0}$. It follows that

$$
\sum_{k \in \mathbf{Z}^{d}}(f * \varphi)(x-\varepsilon k) \psi(\varepsilon k) \varepsilon^{d}
$$

is a Riemann sum which converges to

$$
\int(f * \varphi)(x-y) \psi(y) d y=((f * \varphi) * \psi)(x)
$$

as $\varepsilon>0$ tends to zero. Hence (3.2) holds and the result follows.

The next lemma shows that similar limit properties for convolutions of distributions with mollifiers also work for Gelfand-Shilov distributions and Gelfand-Shilov mollifiers.

Lemma 3.3 Let $s, \sigma>0, \phi, \psi \in \mathcal{S}_{s}^{\sigma}\left(\mathbf{R}^{d}\right)\left(\phi, \psi \in \Sigma_{s}^{\sigma}\left(\mathbf{R}^{d}\right)\right)$ be such that

$$
\int_{\mathbf{R}^{d}} \phi(x) d x=1,
$$

$\phi_{\varepsilon}(x)=\varepsilon^{-d} \phi\left(\varepsilon^{-1} x\right), \varepsilon>0$, and let $f \in\left(\mathcal{S}_{s}^{\sigma}\right)^{\prime}\left(\mathbf{R}^{d}\right)\left(f \in\left(\Sigma_{s}^{\sigma}\right)^{\prime}\left(\mathbf{R}^{d}\right)\right)$. Then the following is true:

(1) $\psi * \phi_{\varepsilon} \rightarrow \psi$ in $\mathcal{S}_{s}^{\sigma}$ (in $\Sigma_{s}^{\sigma}$ ) as $\varepsilon \rightarrow 0+$;

(2) $f * \phi_{\varepsilon} \rightarrow f$ in $\left(\mathcal{S}_{s}^{\sigma}\right)^{\prime}$ (in $\left(\Sigma_{s}^{\sigma}\right)^{\prime}$ ) as $\varepsilon \rightarrow 0+$.

We notice that under the additional assumption

$$
\int_{\mathbf{R}^{d}} x^{\alpha} \phi(x) d x=0, \quad \alpha \in \mathbf{N}^{d} \backslash\{0\},
$$

(1) in Lemma 3.3 is deduced in [7, Proposition 4.1]. By straight-forward modifications of the arguments in the proof of the latter result it also follows that the condition (3.4) may be removed.

In order to be self-contained, we here present a different proof of Lemma 3.3 compared to [7, Proposition 4.1], based on the characterizations of Gelfand-Shilov spaces given in Remark 2.3 and without requiring that (3.4) should hold.

Proof of Lemma 3.3 By duality it suffices to prove (1). We only prove the result in the Roumieu case, i. e. that $\psi * \phi_{\varepsilon} \rightarrow \psi$ in $\mathcal{S}_{s}^{\sigma}$ when $\phi, \psi \in \mathcal{S}_{s}^{\sigma}\left(\mathbf{R}^{d}\right)$. The other 
(Beurling) case follows by similar arguments and is left for the reader.

Let $c \geq 1$ be chosen such that

$$
|x+y|^{\frac{1}{s}} \leq c\left(|x|^{\frac{1}{s}}+|y|^{\frac{1}{s}}\right), \quad x, y \in \mathbf{R}^{d}
$$

and let $r>0$ be chosen such that $\|\phi\|_{(r c, 1)}<\infty$ and $\|\psi\|_{(r c, 1)}<\infty$. (Cf. Remark 2.3 for notations.) By Remark 2.3 and the fact that $\mathscr{F}(f * \psi)=(2 \pi)^{\frac{d}{2}} \widehat{f} \cdot \widehat{\psi}$, the result follows if we prove

$$
\lim _{\varepsilon \rightarrow 0+}\left\|\left(\psi-\psi * \phi_{\varepsilon}\right) e^{\left.r|\cdot|\right|^{\frac{1}{s}}}\right\|_{L^{1}}=0
$$

and

$$
\lim _{\varepsilon \rightarrow 0+} \|\left(\widehat{\psi}\left(1-(2 \pi)^{\frac{d}{2}} \widehat{\phi}(\varepsilon \cdot)\right) e^{\left.r|\cdot|\right|^{\frac{1}{\sigma}}} \|_{L^{1}}=0 .\right.
$$

For any $\varepsilon \in(0,1]$, let

$$
g_{\varepsilon, r}(x, y) \equiv|(\psi(x-\varepsilon y)-\psi(x)) \phi(y)| e^{r|x|^{\frac{1}{s}}}
$$

and

$$
G_{r}(x, y) \equiv|\psi(x) \phi(y)| e^{r\left(|x|^{\frac{1}{s}}+|y|^{\frac{1}{s}}\right)} .
$$

Then

$$
\begin{aligned}
0 & \leq g_{\varepsilon, r}(x, y) \leq|\psi(x-\varepsilon y) \phi(y)| e^{r|x|^{\frac{1}{s}}}+|\psi(x) \phi(y)| e^{r|x|^{\frac{1}{s}}} \\
& \leq|\psi(x-\varepsilon y) \phi(y)| e^{r c\left(|x-\varepsilon y|^{\frac{1}{s}}+\varepsilon|y|^{\frac{1}{s}}\right)}+|\psi(x) \phi(y)| e^{r c\left(|x|^{\frac{1}{s}}+|y|^{\frac{1}{s}}\right)} \\
& \leq G_{c r}(x-\varepsilon y, y)+G_{c r}(x, y) .
\end{aligned}
$$

Here the last inequality follows from the fact that $\varepsilon \leq 1$.

Since $\|\phi\|_{(c r, 1)}+\|\psi\|_{(c r, 1)}<\infty$, it follows that

$$
\begin{aligned}
2\left\|G_{c r}\right\|_{L^{1}} & =\iint_{\mathbf{R}^{2 d}}\left(G_{c r}(x-\varepsilon y, y)+G_{c r}(x, y)\right) d x d y \\
& =2\|\phi\|_{(c r, 1)}\|\psi\|_{(c r, 1)}<\infty .
\end{aligned}
$$

Hence, Lebesgue's theorem gives

$$
\lim _{\varepsilon \rightarrow 0+}\left\|\left(\psi-\psi * \phi_{\varepsilon}\right) e^{r||^{\frac{1}{s}}}\right\|_{L^{1}} \leq \lim _{\varepsilon \rightarrow 0+}\left\|g_{\varepsilon, r}\right\|_{L^{1}}=\left\|\lim _{\varepsilon \rightarrow 0+} g_{\varepsilon, r}\right\|_{L^{1}}=0,
$$

and (3.5) follows.

Since $\int \phi(x) d x=1$ and

$$
\left|\widehat{\psi}(\xi)\left(1-(2 \pi)^{\frac{d}{2}} \widehat{\phi}(\varepsilon \xi)\right)\right| e^{r|\xi|^{\frac{1}{\sigma}}} \leq(2 \pi)^{\frac{d}{2}}\left(1+\|\widehat{\phi}\|_{L^{\infty}}\right)|\widehat{\psi}(\xi)| \in L^{1}\left(\mathbf{R}^{d}\right),
$$


it follows that $\widehat{\phi}(0)=(2 \pi)^{-\frac{d}{2}}$ and that

$$
\begin{aligned}
& \lim _{\varepsilon \rightarrow 0+}\left\|\widehat{\psi}(\xi)\left(1-(2 \pi)^{\frac{d}{2}} \widehat{\phi}(\varepsilon \xi)\right) e^{r|\xi|^{\frac{1}{\sigma}}}\right\|_{L^{1}} \\
& \quad=\left\|\lim _{\varepsilon \rightarrow 0+}\left(\widehat{\psi}(\xi)\left(1-(2 \pi)^{\frac{d}{2}} \widehat{\phi}(\varepsilon \xi)\right) e^{r|\xi|^{\frac{1}{\sigma}}}\right)\right\|_{L^{1}}=0
\end{aligned}
$$

by Lebesgue's theorem. This gives (3.6) and thereby the result.

Remark 3.4 By the previous lemma and Fourier transformations it follows that if $f \in\left(\mathcal{S}_{s}^{\sigma}\right)^{\prime}\left(\mathbf{R}^{d}\right)$ and $\phi, \psi \in \mathcal{S}_{s}^{\sigma}\left(\mathbf{R}^{d}\right)$ fullfil $\phi(0)=1$, then $f \cdot \phi(\varepsilon \cdot) \rightarrow f$ in $\left(\mathcal{S}_{s}^{\sigma}\right)^{\prime}$ and $\psi \cdot \phi(\varepsilon \cdot) \rightarrow \psi$ in $\mathcal{S}_{s}^{\sigma}$ when $\varepsilon \rightarrow 0$. The same holds true with $\Sigma_{s}^{\sigma}$ and $\left(\Sigma_{s}^{\sigma}\right)^{\prime}$ in place of $\mathcal{S}_{s}^{\sigma}$ and $\left(\mathcal{S}_{s}^{\sigma}\right)^{\prime}$ respectively, at each occurrence.

By the previous results it is now straight-forward to prove the following.

Lemma 3.5 Let $\boldsymbol{s}, \boldsymbol{\sigma} \in \mathbf{R}_{+}^{n}, d=d_{1}+\cdots+d_{n}$ and suppose $f \in\left(\mathcal{S}_{s}^{\boldsymbol{\sigma}}\right)^{\prime}\left(\mathbf{R}^{d}\right)$ satisfies $\left\langle f, \varphi_{1} \otimes \cdots \otimes \varphi_{n}\right\rangle=0$ for every $\varphi_{j} \in \mathcal{S}_{s_{j}}^{\sigma_{j}}\left(\mathbf{R}^{d_{j}}\right), j=1, \ldots, n$. Then $f=0$.

The same holds true if each $\mathcal{S}_{s_{j}}^{\sigma_{j}},\left(\mathcal{S}_{s_{j}}^{\sigma_{j}}\right)^{\prime}, \mathcal{S}_{s}^{\sigma}$ and $\left(\mathcal{S}_{s}^{\sigma}\right)^{\prime}$ are replaced by $\Sigma_{s_{j}}^{\sigma_{j}},\left(\Sigma_{s_{j}}^{\sigma_{j}}\right)^{\prime}$, $\Sigma_{s}^{\sigma}$ and $\left(\Sigma_{s}^{\sigma}\right)^{\prime}$, respectively, at each occurrence.

Proof We only prove the result in the Roumieu case. The Beurling case follows by similar arguments and is left for the reader. We use the same notations as in the previous proofs.

First suppose that $n=2$. Let $\varphi \in \mathcal{S}_{S_{1}, s_{2}}^{\sigma_{1}, \sigma_{2}}\left(\mathbf{R}^{d_{1}+d_{2}}\right), \phi_{j} \in \mathcal{S}_{S_{j}}^{\sigma_{j}}\left(\mathbf{R}^{d_{j}}\right)$ be such that

$$
\int_{\mathbf{R}^{d_{j}}} \phi_{j}\left(x_{j}\right) d x_{j}=1
$$

and let

$$
\phi_{\varepsilon}=\varepsilon^{-\left(d_{1}+d_{2}\right)}\left(\phi_{1} \otimes \phi_{2}\right)\left(\varepsilon^{-1} \cdot\right),
$$

when $\varepsilon>0$ is real. Then the assumptions imply that $\check{f} * \phi_{\varepsilon}=0$ for every $\varepsilon$. Here $\check{f}$ is defined by $\check{f}(x)=f(-x)$. By Lemmas 3.2 and 3.3 we get

$$
\langle f, \varphi\rangle=\lim _{\varepsilon \rightarrow 0+}\left\langle f, \phi_{\varepsilon} * \varphi\right\rangle=\lim _{\varepsilon \rightarrow 0+}\left(\check{f} *\left(\phi_{\varepsilon} * \varphi\right)\right)(0)=\lim _{\varepsilon \rightarrow 0+}\left(\left(\check{f} * \phi_{\varepsilon}\right) * \varphi\right)(0)=0,
$$

and the result follows for $n=2$.

For general $n \geq 2$, the result follows from the case $n=2$ and induction. The details are left for the reader.

Proof of Theorem 1.2 We only prove the result in the Roumieu cases. The Beurling cases follow by similar arguments and are left for the reader.

By straight-forward computations it follows that

$$
\varphi \mapsto\left\langle f_{1}, \psi_{1}\right\rangle \quad \text { with } \quad \psi_{1}\left(x_{1}\right)=\left\langle f_{2}, \varphi\left(x_{1}, \cdot\right)\right\rangle,
$$

and 


$$
\varphi \mapsto\left\langle f_{2}, \psi_{2}\right\rangle, \quad \text { with } \quad \psi_{2}\left(x_{2}\right)=\left\langle f_{1}, \varphi\left(\cdot, x_{2}\right)\right\rangle \text {, }
$$

define continuous linear forms $g_{1}$ and $g_{2}$ on $\mathcal{S}_{s_{1}, s_{2}}^{\sigma_{1} \sigma_{2}}\left(\mathbf{R}^{d_{1}+d_{2}}\right)$. Hence $g_{1}, g_{2} \in\left(\mathcal{S}_{s_{1}, s_{2}}^{\sigma_{1}, \sigma_{2}}\right)^{\prime}\left(\mathbf{R}^{d_{1}+d_{2}}\right)$. It is obvious that both $g_{1}$ and $g_{2}$ in place of $f$ satisfy (1.2), and the existence of $f$ follows.

If $f \in\left(\mathcal{S}_{S_{1}, s_{2}}^{\sigma_{1}, \sigma_{2}}\right)^{\prime}\left(\mathbf{R}^{d_{1}+d_{2}}\right)$ is arbitrary such that (1.2) holds, then

$$
\left\langle f-g_{j}, \varphi_{1} \otimes \varphi_{2}\right\rangle=\left\langle f_{1}, \varphi_{1}\right\rangle\left\langle f_{2}, \varphi_{2}\right\rangle-\left\langle f_{1}, \varphi_{1}\right\rangle\left\langle f_{2}, \varphi_{2}\right\rangle=0,
$$

and Lemma 3.5 shows that $f=g_{1}=g_{2}$. This gives the uniqueness of $f$, as well as (1.3).

In order to consider corresponding multi-linear situation of Theorem 1.2, we let $S_{n}$ be the permutation group of $\{1, \ldots, n\}$, and let inductively

$$
\varphi_{n, \tau}\left(x_{\tau(1)}, \ldots, x_{\tau(n)}\right)=\varphi\left(x_{1}, \ldots, x_{n}\right), \quad x_{j} \in \mathbf{R}^{d_{j}}, \tau \in S_{n},
$$

and

$$
\varphi_{j, \tau}\left(x_{\tau(1)}, \ldots, x_{\tau(j)}\right)=\left\langle f_{\tau(j+1)}, \varphi_{j+1, \tau}\left(x_{\tau(1)}, \ldots, x_{\tau(j)}, \cdot\right)\right\rangle
$$

when $f_{j}$ for $j=1, \ldots, n$ are suitable (ultra-)distributions and $\varphi$ is a suitable function. Also set

$$
\begin{aligned}
& \boldsymbol{s}_{j, \tau}=\left(s_{\tau(1)}, \ldots, s_{\tau(j)}\right), \quad \boldsymbol{\sigma}_{j, \tau}=\left(\sigma_{\tau(1)}, \ldots, \sigma_{\tau(j)}\right) \\
& \text { and } \quad d_{j, \tau}=d_{\tau(1)}+\cdots+d_{\tau(j)},
\end{aligned}
$$

when $j=1, \ldots, n$ and $s, \boldsymbol{\sigma} \in \mathbf{R}_{+}^{n}$. Then Theorem 1.2 can be reformulated as follows.

Theorem 3.6 Let $\tau \in S_{2}, d=d_{1}+d_{2}, \boldsymbol{s}, \boldsymbol{\sigma} \in \mathbf{R}_{+}^{2}, d_{j, \tau}, \boldsymbol{s}_{j, \tau}$ and $\boldsymbol{\sigma}_{j, \tau}$ be as in (3.9), $f_{j} \in\left(\mathcal{S}_{s_{j}}^{\sigma_{j}}\right)^{\prime}\left(\mathbf{R}^{d_{j}}\right), \varphi \in \mathcal{S}_{\boldsymbol{s}}^{\sigma}\left(\mathbf{R}^{d}\right)$ and let $\varphi_{j, \tau}$ be given by (3.7) and (3.8), $j=1,2$. Then $\varphi_{j, \tau} \in \mathcal{S}_{\boldsymbol{s}_{j \tau}}^{\boldsymbol{\sigma}_{j, \tau}}\left(\mathbf{R}^{d_{j, \tau}}\right)$, and there is a unique distribution $f$ in $\left(\mathcal{S}_{\boldsymbol{s}}^{\boldsymbol{\sigma}}\right)^{\prime}\left(\mathbf{R}^{d}\right)$ such that for every $\varphi_{j} \in \mathcal{S}_{s_{j}}^{\sigma_{j}}\left(\mathbf{R}^{d_{j}}\right), j=1,2$,

$$
\left\langle f, \varphi_{1} \otimes \varphi_{2}\right\rangle=\prod_{k=1}^{2}\left\langle f_{k}, \varphi_{k}\right\rangle \quad \text { and } \quad\langle f, \varphi\rangle=\left\langle f_{\tau(1)}, \varphi_{1, \tau}\right\rangle
$$

hold.

The same holds true with $\Sigma_{s_{j}}^{\sigma_{j}},\left(\Sigma_{s_{j}}^{\sigma_{j}}\right)^{\prime}, \Sigma_{s}^{\sigma}$ and $\left(\Sigma_{s}^{\sigma}\right)^{\prime}$ in place of $\mathcal{S}_{s_{j}}^{\sigma_{j}},\left(\mathcal{S}_{s_{j}}^{\sigma_{j}}\right)^{\prime}, \mathcal{S}_{s}^{\sigma}$ and $\left(\mathcal{S}_{s}^{\sigma}\right)^{\prime}$, respectively, at each occurrence.

Here the second equality in (3.10) is the same as the Fubini property (1.3). The multi-linear version of the previous theorem is the following, and follows by similar arguments as for the proof of Theorem 3.6. The details are left for the reader.

Theorem 3.6' Let $\tau \in S_{n}, d=d_{1}+\ldots+d_{n}, \boldsymbol{s}, \boldsymbol{\sigma} \in \mathbf{R}_{+}^{n}, d_{j, \tau}, \boldsymbol{s}_{j, \tau}$ and $\boldsymbol{\sigma}_{j, \tau}$ be as in (3.9), $f_{j} \in\left(\mathcal{S}_{s_{j}}^{\sigma_{j}}\right)^{\prime}\left(\mathbf{R}^{d_{j}}\right), \varphi \in \mathcal{S}_{\boldsymbol{s}}^{\sigma}\left(\mathbf{R}^{d}\right)$ and let $\varphi_{j, \tau}$ be given by (3.7) and (3.8), 
$j=1, \ldots, n$. Then $\varphi_{j, \tau} \in \mathcal{S}_{\boldsymbol{s}_{j \tau}}^{\boldsymbol{\sigma}_{j, \tau}}\left(\mathbf{R}^{d_{j, \tau}}\right)$, and there is a unique distribution $f$ in $\left(\mathcal{S}_{s}^{\sigma}\right)^{\prime}\left(\mathbf{R}^{d}\right)$ such that for every $\varphi_{j} \in \mathcal{S}_{S_{j}}^{\sigma_{j}}\left(\mathbf{R}^{d_{j}}\right), j=1, \ldots, n$,

$$
\left\langle f, \varphi_{1} \otimes \cdots \otimes \varphi_{n}\right\rangle=\prod_{k=1}^{n}\left\langle f_{k}, \varphi_{k}\right\rangle \quad \text { and } \quad\langle f, \varphi\rangle=\left\langle f_{\tau(1)}, \varphi_{1, \tau}\right\rangle
$$

hold.

The same holds true with $\Sigma_{s_{j}}^{\sigma_{j}},\left(\Sigma_{s_{j}}^{\sigma_{j}}\right)^{\prime}, \Sigma_{s}^{\sigma}$ and $\left(\Sigma_{s}^{\sigma}\right)^{\prime}$ in place of $\mathcal{S}_{s_{j}}^{\sigma_{j}},\left(\mathcal{S}_{s_{j}}^{\sigma_{j}}\right)^{\prime}, \mathcal{S}_{s}^{\sigma}$ and $\left(\mathcal{S}_{s}^{\sigma}\right)^{\prime}$, respectively, at each occurrence.

Example 3.7 Let $s, \sigma>0$. An important object in time-frequency and micro-local analysis concerns the short-time Fourier transform (cf. e.g., [15]). If $\phi \in$ $\mathcal{S}_{s}^{\sigma}\left(\mathbf{R}^{d}\right) \backslash\{0\}$ is fixed, then the short-time Fourier transform of $f \in \mathcal{S}_{s}^{\sigma}\left(\mathbf{R}^{d}\right)$ is defined by

$$
V_{\phi} f(x, \xi)=(2 \pi)^{-\frac{d}{2}} \int_{\mathbf{R}^{d}} f(y) \overline{\phi(y-x)} e^{-i\langle y, \xi\rangle} d y .
$$

It follows that

$$
V_{\phi} f(x, \xi)=(2 \pi)^{-\frac{d}{2}}\left\langle f, \overline{\phi(\cdot-x)} e^{-i\langle\cdot, \xi\rangle}\right\rangle
$$

and

$$
V_{\phi} f(x, \xi)=\mathscr{F}(f \cdot \overline{\phi(\cdot-x)})(\xi)
$$

for such choices of $\phi$ and $f$.

We notice that the right-hand side of (3.11) also makes sense as a smooth function on $\mathbf{R}^{2 d}$ if the assumption on $f$ is relaxed into $f \in\left(\mathcal{S}_{s}^{\sigma}\right)^{\prime}\left(\mathbf{R}^{d}\right)$. For such $f$ we therefore let (3.11) define the short-time Fourier transform of $f$ with respect to $\phi$. Since the map which takes $\phi$ into $y \mapsto \phi(y-x) e^{i\langle y, \xi\rangle}$ is continuous and smooth with respect to $(x, \xi)$ from $\mathcal{S}_{s}^{\sigma}\left(\mathbf{R}^{d}\right)$ to itself it follows that $V_{\phi} f$ is smooth. By [16, Proposition 2.2] it follows that $V_{\phi} f$ belongs to $\left(\mathcal{S}_{s, \sigma}^{\sigma, s}\right)^{\prime}\left(\mathbf{R}^{2 d}\right)$. Consequently,

$$
V_{\phi} f \in\left(\mathcal{S}_{s, \sigma}^{\sigma, s}\right)^{\prime}\left(\mathbf{R}^{2 d}\right) \cap C^{\infty}\left(\mathbf{R}^{2 d}\right) .
$$

Let $U$ be the operator which takes any $F(x, y)$ into $F(y, y-x)$ and recall that $\mathscr{F}_{2} F$ is the partial Fourier transform of $F(x, y)$ with respect to the $y$ variable. Then the righthand side of (3.12) equals

$$
\left(\mathscr{F}_{2}(U(f \otimes \bar{\phi}))\right)(x, \xi) .
$$

We notice that the right-hand side makes sense as an element in $\left(\mathcal{S}_{s, \sigma}^{\sigma, s}\right)^{\prime}\left(\mathbf{R}^{2 d}\right)$ for any $f, \phi \in\left(\mathcal{S}_{s}^{\sigma}\right)^{\prime}\left(\mathbf{R}^{d}\right)$ in view of Remark 2.2, which may be used to extend the definition of the short-time Fourier transform to even more general situations. 
We claim that the right-hand sides of (3.11) and (3.12) agree when $f \in\left(\mathcal{S}_{s}^{\sigma}\right)^{\prime}\left(\mathbf{R}^{d}\right)$ and $\phi \in \mathcal{S}_{s}^{\sigma}\left(\mathbf{R}^{d}\right)$. In particular, by letting $s=\sigma$, we recover (A.1) in [4, Appendix].

In fact, let $\psi \in \mathcal{S}_{s, \sigma}^{\sigma, s}\left(\mathbf{R}^{2 d}\right)$ and set

$$
\begin{aligned}
\varphi(x, \xi, y) & \equiv \psi(x, \xi) \overline{\phi(y-x)} e^{-i\langle y, \xi\rangle} \in \mathcal{S}_{s, \sigma, s}^{\sigma, s}\left(\mathbf{R}^{3 d}\right), \\
F & \equiv 1_{\mathbf{R}^{2 d}} \otimes f=1_{\mathbf{R}^{d}} \otimes 1_{\mathbf{R}^{d}} \otimes f \in\left(\mathcal{S}_{s, \sigma, s}^{\sigma, s, \sigma}\right)^{\prime}\left(\mathbf{R}^{3 d}\right), \\
\varphi_{1}(x, y) & \equiv \int_{\mathbf{R}^{d}} \varphi(x, \xi, y) d \xi=\int_{\mathbf{R}^{d}} \psi(x, \xi) \overline{\phi(y-x)} e^{-i\langle y, \xi\rangle} d x d \xi, \\
\varphi_{2}(x, \xi) & \equiv\left\langle f, \psi(x, \xi) \overline{\phi(\cdot-x)} e^{-i\langle\cdot, \xi\rangle}\right\rangle=\psi(x, \xi) \cdot V_{\phi} f(x, \xi),
\end{aligned}
$$

and let $g$ be the right-hand side of (3.12). By the Fubini property at the right-hand of $(3.10)^{\prime}$ we get

$$
\langle F, \varphi\rangle=\left\langle 1_{\mathbf{R}^{d}} \otimes f, \varphi_{1}\right\rangle=\langle g, \psi\rangle
$$

and

$$
\langle F, \varphi\rangle=\left\langle 1_{\mathbf{R}^{2 d}}, \varphi_{2}\right\rangle=\left\langle V_{\phi} f, \psi\right\rangle .
$$

Since $\psi$ was arbitrarily chosen, it follows that $g=V_{\phi} f$ in $\left(\mathcal{S}_{s, \sigma}^{\sigma, s}\right)^{\prime}\left(\mathbf{R}^{2 d}\right)$, and the claim follows.

Remark 3.8 By straight-forward modifications of the arguments in the previous example it also follows that the right-hand sides of (3.11) and (3.12) agree when $f \in\left(\Sigma_{s}^{\sigma}\right)^{\prime}\left(\mathbf{R}^{d}\right)$ and $\phi \in \Sigma_{s}^{\sigma}\left(\mathbf{R}^{d}\right)$.

\section{Tensor product of Pilipović spaces}

In this section we discuss the tensor products of Pilipovic spaces. Especially we prove Theorem 1.2. Thereafter we deduce a multi-linear version of this result.

First we show that the tensor products take Pilipović spaces into Pilipović spaces.

Proposition 4.1 Let $s \in \overline{\mathbf{R}_{b}}$. Then the following is true:

(1) the map $\left(f_{1}, f_{2}\right) \mapsto f_{1} \otimes f_{2}$ from $\mathscr{S}\left(\mathbf{R}^{d_{1}}\right) \times \mathscr{S}\left(\mathbf{R}^{d_{2}}\right)$ to $\mathscr{S}\left(\mathbf{R}^{d_{1}+d_{2}}\right)$, restricts to a continuous map from $\mathcal{H}_{s}\left(\mathbf{R}^{d_{1}}\right) \times \mathcal{H}_{s}\left(\mathbf{R}^{d_{2}}\right)$ to $\mathcal{H}_{s}\left(\mathbf{R}^{d_{1}+d_{2}}\right)$;

(2) the map $\left(f_{1}, f_{2}\right) \mapsto f_{1} \otimes f_{2}$ from $\mathscr{S}\left(\mathbf{R}^{d_{1}}\right) \times \mathscr{S}\left(\mathbf{R}^{d_{2}}\right)$ to $\mathscr{S}\left(\mathbf{R}^{d_{1}+d_{2}}\right)$, restricts to a continuous map from $\mathcal{H}_{0, s}\left(\mathbf{R}^{d_{1}}\right) \times \mathcal{H}_{0, s}\left(\mathbf{R}^{d_{2}}\right)$ to $\mathcal{H}_{0, s}\left(\mathbf{R}^{d_{1}+d_{2}}\right)$.

Proof We only prove (1) and in the case $s>0$. The assertion (2) and the case $s=0$ in (1) follow by similar arguments and are left for the reader. If

$$
f_{j}=\sum_{\alpha_{j} \in \mathbf{N}^{d_{j}}} c_{h}\left(f_{j}, \alpha_{j}\right) h_{\alpha_{j}}, \quad j=1,2,
$$

and $f=f_{1} \otimes f_{2}$, then 


$$
c_{h}(f, \alpha)=c_{h}\left(f_{1}, \alpha_{1}\right) c_{h}\left(f_{2}, \alpha_{2}\right), \quad \alpha=\left(\alpha_{1}, \alpha_{2}\right), \alpha_{j} \in \mathbf{N}^{d_{j}}, j=1,2 .
$$

If $s \in \mathbf{R}_{+}$, then

$$
\left|c_{h}\left(f_{j}, \alpha_{j}\right)\right| \lesssim e^{-r\left|\alpha_{j}\right|^{\frac{1}{2 s}}}
$$

for some $r>0$. This gives

$$
\left|c_{h}(f, \alpha)\right| \lesssim e^{-r\left(\left|\alpha_{1}\right|^{\frac{1}{2 s}}+\left|\alpha_{2}\right|^{\frac{1}{2 s}}\right)} \leq e^{-r|\alpha|^{\frac{1}{2 s}} /\left(1+2^{\frac{1}{s}}\right)}, \quad \alpha=\left(\alpha_{1}, \alpha_{2}\right),
$$

and it follows that $f \in \mathcal{H}_{s}\left(\mathbf{R}^{d}\right)$.

If instead $s=b_{\sigma}$, for some $\sigma>0$, then

$$
\left|c_{h}\left(f_{j}, \alpha_{j}\right)\right| \lesssim r^{\left|\alpha_{j}\right|} \alpha_{j} !^{-\frac{1}{2 \sigma}}, \quad j=1,2,
$$

for some $r>0$. Hence, if $\alpha=\left(\alpha_{1}, \alpha_{2}\right)$, we get

$$
\left|c_{h}\left(f_{1} \otimes f_{2}, \alpha\right)\right| \lesssim r^{|\alpha|}\left(\alpha_{1} ! \alpha_{2} !\right)^{-\frac{1}{2 \sigma}}=r^{|\alpha|} \alpha !^{-\frac{1}{2 \sigma}}
$$

for some $r>0$, and it follows that $f \in \mathcal{H}_{s}\left(\mathbf{R}^{d}\right)$ in this case as well. This shows that the map $\left(f_{1}, f_{2}\right) \rightarrow f_{1} \otimes f_{2}$ is continuous from $\mathcal{H}_{s}\left(\mathbf{R}^{d_{1}}\right) \times \mathcal{H}_{s}\left(\mathbf{R}^{d_{2}}\right)$ to $\mathcal{H}_{s}\left(\mathbf{R}^{d_{1}+d_{2}}\right)$, and the result follows.

The uniqueness assertions of Theorem 1.3 follow from the following lemma.

Lemma 4.2 Let $s \in \overline{\mathbf{R}_{b}}, d=d_{1}+\cdots+d_{n}$ and suppose $f \in \mathcal{H}_{s}^{\prime}\left(\mathbf{R}^{d}\right)$ satisfies $\left\langle f, \varphi_{1} \otimes \cdots \otimes \varphi_{n}\right\rangle=0$ for every $\varphi_{j} \in \mathcal{H}_{s}\left(\mathbf{R}^{d_{j}}\right), j=1, \ldots, n$. Then $f=0$.

The same holds true if each $\mathcal{H}_{s}$ and $\mathcal{H}_{s}^{\prime}$ are replaced by $\mathcal{H}_{0, s}$ and $\mathcal{H}_{0, s}^{\prime}$, respectively.

Proof Let $c_{h}(f, \alpha)$ be the Hermite coefficient of $f$ of order $\alpha=\left(\alpha_{1}, \ldots, \alpha_{n}\right)$, where $\alpha_{j} \in \mathbf{N}^{d_{j}}$. By choosing $\varphi_{j}=h_{\alpha_{j}}$, we get

$$
0=\left\langle f, h_{\alpha_{1}} \otimes \cdots \otimes h_{\alpha_{n}}\right\rangle=\left\langle f, h_{\alpha}\right\rangle=c_{h}(f, \alpha),
$$

giving that $f=0$.

Proof of Theorem 1.3 Let $d=d_{1}+d_{2}$. We shall deal with the Hermite sequence representations of the elements in the Pilipović spaces. Such approach is performed in [14], when deducing tensor product and kernel results for tempered distributions. We only prove the results when $f_{j} \in \mathcal{H}_{s}^{\prime}\left(\mathbf{R}^{d_{j}}\right)$ and $s>0$. The cases when $f_{j} \in$ $\mathcal{H}_{0, s}^{\prime}\left(\mathbf{R}^{d_{j}}\right)$ or $s=0$ follow by similar arguments and are left for the reader.

The uniqueness follows from Lemma 4.2. We need to prove the existence of $f$ which fullfils the asserted properties.

We have

$$
f_{j}=\sum_{\alpha_{j} \in \mathbf{N}^{d_{j}}} c_{h}\left(f_{j}, \alpha_{j}\right) h_{\alpha_{j}}
$$


where $c_{h}\left(f_{j}, \alpha_{j}\right)$ for every $\alpha_{j} \in \mathbf{N}^{d_{j}}$ are unique and equal to $\left(f_{j}, h_{\alpha_{j}}\right), j=1,2$.

Now let $f$ be the element in $\mathcal{H}_{0}^{\prime}\left(\mathbf{R}^{d}\right), d=d_{1}+d_{2}$ with expansion

$$
f=\sum_{\alpha \in \mathbf{N}^{d}} c_{h}(f, \alpha) h_{\alpha}
$$

where

$$
c_{h}(f, \alpha)=c_{h}\left(f_{1}, \alpha_{1}\right) c_{h}\left(f_{2}, \alpha_{2}\right), \quad \alpha=\left(\alpha_{1}, \alpha_{2}\right), \alpha_{j} \in \mathbf{N}^{d_{j}}, j=1,2 .
$$

We claim that $f \in \mathcal{H}_{s}^{\prime}\left(\mathbf{R}^{d}\right)$.

In fact, if $s \in \mathbf{R}_{+}$, then

$$
\left|c_{h}\left(f_{j}, \alpha_{j}\right)\right| \lesssim e^{\varepsilon\left|\alpha_{j}\right|^{\frac{1}{2 s}}}
$$

for every $\varepsilon>0$, and it follows that

$$
\left|c_{h}(f, \alpha)\right| \lesssim e^{\varepsilon\left(\left|\alpha_{1}\right|^{\frac{1}{2 s}}+\left|\alpha_{2}\right|^{\frac{1}{2 s}}\right)} \leq e^{2 \varepsilon|\alpha|^{\frac{1}{2 s}}}, \quad \alpha=\left(\alpha_{1}, \alpha_{2}\right),
$$

for every $\varepsilon>0$. This is the same as $f \in \mathcal{H}_{s}^{\prime}\left(\mathbf{R}^{d}\right)$.

If instead $s=b_{\sigma}$, for some $\sigma>0$, then

$$
\left|c_{h}\left(f_{j}, \alpha_{j}\right)\right| \lesssim r^{\left|\alpha_{j}\right|} \alpha_{j} !^{\frac{1}{2 \sigma}}, \quad j=1,2,
$$

for every $r>0$. Hence, if $\alpha=\left(\alpha_{1}, \alpha_{2}\right)$, we get

$$
\left|c_{h}(f, \alpha)\right| \lesssim r^{|\alpha|}\left(\alpha_{1} ! \alpha_{2} !\right)^{\frac{1}{2 \sigma}}=r^{|\alpha|} \alpha !^{\frac{1}{2 \sigma}},
$$

for every $r>0$, and it follows that $f \in \mathcal{H}_{s}^{\prime}\left(\mathbf{R}^{d}\right)$ in this case as well.

If $\varphi_{j} \in \mathcal{H}_{0}\left(\mathbf{R}^{d_{j}}\right)$ and $\varphi \in \mathcal{H}_{0}\left(\mathbf{R}^{d}\right), j=1,2$, then (1.2) and (1.3) follow by straight-forward computations, using the fact that the set of Hermite functions is an orthonormal basis of $L^{2}$. For general $\varphi_{j} \in \mathcal{H}_{s}\left(\mathbf{R}^{d_{j}}\right)$ and $\varphi \in \mathcal{H}_{s}\left(\mathbf{R}^{d}\right), j=1,2$, the result now follows from dominating convergence, using the fact that $\mathcal{H}_{0}\left(\mathbf{R}^{d}\right)$ is dense in $\mathcal{H}_{s}\left(\mathbf{R}^{d}\right)$.

In order to formulate a multi-linear version of Theorem 1.3 we first reformulate the result as follows.

Theorem 4.3 Let $\tau \in S_{2}, d=d_{1}+d_{2}, s \in \overline{\mathbf{R}_{b}}, d_{j, \tau}$ be as in (3.9), $f_{j} \in\left(\mathcal{H}_{s}\right)^{\prime}\left(\mathbf{R}^{d_{j}}\right)$, $\varphi \in \mathcal{H}_{s}\left(\mathbf{R}^{d}\right)$ and let $\varphi_{j, \tau}$ be given by (3.7) and (3.8), $j=1,2$. Then $\varphi_{j, \tau} \in \mathcal{H}_{s}\left(\mathbf{R}^{d_{j, \tau}}\right)$, and there is a unique distribution $f$ in $\mathcal{H}_{s}^{\prime}\left(\mathbf{R}^{d}\right)$ such that for every $\varphi_{j} \in \mathcal{H}_{s}\left(\mathbf{R}^{d_{j}}\right)$, $j=1,2$,

$$
\left\langle f, \varphi_{1} \otimes \varphi_{2}\right\rangle=\prod_{k=1}^{2}\left\langle f_{k}, \varphi_{k}\right\rangle \quad \text { and } \quad\langle f, \varphi\rangle=\left\langle f_{\tau(1)}, \varphi_{1, \tau}\right\rangle
$$

hold. 
The same holds true with $\mathcal{H}_{0, s}$ and $\mathcal{H}_{0, s}^{\prime}$ in place of $\mathcal{H}_{s}$ and $\mathcal{H}_{s}^{\prime}$, respectively, at each occurrence.

The multi-linear version of the previous theorem is the following, and follows from the previous result and induction. The details are left for the reader.

Theorem 4.3' Let $\tau \in S_{n}, \quad d=d_{1}+\cdots+d_{n}, \quad s \in \overline{\mathbf{R}_{b}}, \quad d_{j, \tau}$ be as in (3.9), $f_{j} \in\left(\mathcal{H}_{s}\right)^{\prime}\left(\mathbf{R}^{d_{j}}\right), \varphi \in \mathcal{H}_{s}\left(\mathbf{R}^{d}\right)$ and let $\varphi_{j, \tau}$ be given by (3.7) and (3.8), $j=1, \ldots, n$. Then $\varphi_{j, \tau} \in \mathcal{H}_{s}\left(\mathbf{R}^{d_{j, \tau}}\right)$, and there is a unique distribution $f$ in $\mathcal{H}_{s}^{\prime}\left(\mathbf{R}^{d}\right)$ such that for every $\varphi_{j} \in \mathcal{H}_{s}\left(\mathbf{R}^{d_{j}}\right), j=1, \ldots, n$,

$$
\left\langle f, \varphi_{1} \otimes \cdots \otimes \varphi_{n}\right\rangle=\prod_{k=1}^{n}\left\langle f_{k}, \varphi_{k}\right\rangle \quad \text { and } \quad\langle f, \varphi\rangle=\left\langle f_{\tau(1)}, \varphi_{1, \tau}\right\rangle
$$

hold.

The same holds true with $\mathcal{H}_{0, s}$ and $\mathcal{H}_{0, s}^{\prime}$ in place of $\mathcal{H}_{s}$ and $\mathcal{H}_{s}^{\prime}$, respectively, at each occurrence.

Remark 4.4 Only certain parts of the properties in Example 3.7 carry over to Pilipović spaces of functions and distributions, in the case when these spaces do not agree with Gelfand-Shilov spaces of functions and distributions. (See Remark 2.5). In order to deal with such questions, it it convenient to consider the image of such spaces under the Bargmann transform, which is defined by

$$
\left(\mathfrak{B}_{d} f\right)(z)=\pi^{-\frac{d}{4}}\left\langle f, \exp \left(-\frac{1}{2}\left(\langle z, z\rangle+|\cdot|^{2}\right)+\sqrt{2}\langle z, \cdot\rangle\right)\right\rangle,
$$

when $f$ is a suitable (ultra-)distribution on $\mathbf{R}^{d}$ (cf. [1, 16]). Here

$$
\langle z, z\rangle=\sum_{j=1}^{d} z_{j}^{2}, \quad z=\left(z_{1}, \ldots, z_{d}\right) \in \mathbf{C}^{d} .
$$

In fact, let $\mathcal{A}_{s}\left(\mathbf{C}^{d}\right)\left(\mathcal{A}_{0, s}\left(\mathbf{C}^{d}\right)\right)$ be the set of all $F$ in $A\left(\mathbf{C}^{d}\right)$, the set of entire functions on $\mathbf{C}^{d}$, which satisfies

$$
|F(z)| \lesssim e^{r(\log \langle z\rangle)^{\frac{1}{1-2 s}}}
$$

when $s<\frac{1}{2}$ and

$$
|F(z)| \lesssim e^{r|z|^{\frac{2 \sigma}{\sigma+1}}}
$$

when $s=b_{\sigma}$, for some $r>0$ (for every $r>0$ ). Here $\langle z\rangle=\left(1+|z|^{2}\right)^{\frac{1}{2}}$ when $z \in \mathbf{Z}^{d}$. Also let $\mathcal{A}_{0,1 / 2}\left(\mathbf{C}^{d}\right)$ be the set of all $F \in A\left(\mathbf{C}^{d}\right)$ such that $|F(z)| \lesssim e^{r|z|^{2}}$ for all $r>0$. Then it is proved in $[6,16]$ that $\mathfrak{B}_{d}$ is bijective from $\mathcal{H}_{s}\left(\mathbf{R}^{d}\right)$ to $\mathcal{A}_{s}\left(\mathbf{C}^{d}\right)$ when $s \in \mathbf{R}_{b}$ and $s<\frac{1}{2}$, and from $\mathcal{H}_{0, s}\left(\mathbf{R}^{d}\right)$ to $\mathcal{A}_{0, s}\left(\mathbf{C}^{d}\right)$ when $s \in \mathbf{R}_{b}$ and $s \leq \frac{1}{2}$.

By straight-forward computations we have for every $x_{0} \in \mathbf{R}^{d}$ that 


$$
\left(\mathfrak{V}_{d}\left(f\left(\cdot-x_{0}\right)\right)\right)(z)=e^{\sqrt{2}\left\langle z, x_{0}\right\rangle+\frac{1}{2}\left|x_{0}\right|^{2}}\left(\mathfrak{V}_{d} f\right)\left(z+\sqrt{2} x_{0}\right)
$$

and

$$
\left(\mathfrak{B}_{d}\left(f e^{-i\left\langle\cdot, \xi_{0}\right\rangle}\right)\right)(z)=e^{-\sqrt{2} i\left\langle z, \xi_{0}\right\rangle+\frac{1}{2}\left|\xi_{0}\right|^{2}}\left(\mathfrak{B}_{d} f\right)\left(z+i \sqrt{2} \xi_{0}\right) .
$$

Consequently, by Remark 2.5 and the mapping properties of the Pilipović spaces above under the Bargmann transform, it follows that the following is true:

(1) $\mathcal{H}_{s}\left(\mathbf{R}^{d}\right)$ and $\mathcal{H}_{s}^{\prime}\left(\mathbf{R}^{d}\right)$ are invariant under translations and modulations, if and only if $s \geq b_{1}$;

(2) $\mathcal{H}_{0, s}\left(\mathbf{R}^{d}\right)$ and $\mathcal{H}_{0, s}^{\prime}\left(\mathbf{R}^{d}\right)$ are invariant under translations and modulations, if and only if $s>b_{1}$.

In particular, the short-time Fourier transform

$$
V_{\phi} f(x, \xi)=\left\langle f, \overline{\phi(\cdot-x)} e^{-i\langle\cdot, \xi\rangle}\right\rangle
$$

makes sense as a smooth function when $s \geq b_{1}, f \in \mathcal{H}_{s}^{\prime}\left(\mathbf{R}^{d}\right)$ and $\phi \in \mathcal{H}_{s}\left(\mathbf{R}^{d}\right)$, or when $s>b_{1}, f \in \mathcal{H}_{0, s}^{\prime}\left(\mathbf{R}^{d}\right)$ and $\phi \in \mathcal{H}_{0, s}\left(\mathbf{R}^{d}\right)$.

On the other hand, for $s<\frac{1}{2}$, it seems to be difficult to guarantee that (3.12) is true in general, since the map $U$ in Example 3.7 seems not to be well-defined on Pilipović spaces which fail to be Gelfand-Shilov spaces.

Remark 4.5 It is observed already in [1] that if

$$
e_{\alpha}(z)=\frac{z^{\alpha}}{\sqrt{\alpha !}}, \quad z \in \mathbf{C}^{d}, \alpha \in \mathbf{N}^{d},
$$

then $\mathfrak{B}_{d} h_{\alpha}=e_{\alpha}$. For any expansion $f$ in (2.5), we define the Bargmann transform of $f$ as the formal power series expansion

$$
F(z)=\sum_{\alpha \in \mathbf{N}^{d}} c_{A}(F, \alpha) e_{\alpha}(z),
$$

with

$$
c_{A}(F, \alpha)=c_{h}(f, \alpha)
$$

For any $s \in \overline{\mathbf{R}}_{b}$, we also let

$$
\mathcal{A}_{0, s}\left(\mathbf{C}^{d}\right), \quad \mathcal{A}_{s}\left(\mathbf{C}^{d}\right), \quad \mathcal{A}_{0, s}^{\prime}\left(\mathbf{C}^{d}\right) \quad \text { and } \quad \mathcal{A}_{0, s}^{\prime}\left(\mathbf{C}^{d}\right)
$$

be the images of

$$
\mathcal{H}_{0, s}\left(\mathbf{R}^{d}\right), \quad \mathcal{H}_{s}\left(\mathbf{R}^{d}\right), \quad \mathcal{H}_{0, s}^{\prime}\left(\mathbf{R}^{d}\right) \quad \text { and } \quad \mathcal{H}_{0, s}^{\prime}\left(\mathbf{R}^{d}\right),
$$

respectively, under the Bargmann transform. We also let the topologies of the former spaces be inherited from the latter spaces. Let $d \lambda(z)$ be the Lebesgue measure on $\mathbf{C}^{d}, d \mu(z)$ be the Gauss measure given by $d \mu(z)=\pi^{-d} e^{-|z|^{2}} d \lambda(z)$ and let $A^{2}\left(\mathbf{C}^{d}\right)$ be the Hilbert space of all $F \in A\left(\mathbf{C}^{d}\right)$ such that 


$$
\|F\|_{A^{2}} \equiv\left(\int_{\mathbf{C}^{d}}|F(z)|^{2} d \mu(z)\right)^{\frac{1}{2}}
$$

is finite. Then the scalar product of $A^{2}\left(\mathbf{C}^{d}\right)$ is given by

$$
(F, G)_{A^{2}}=\int_{\mathbf{C}^{d}} F(z) \overline{G(z)} d \mu(z), \quad F, G \in A^{2}\left(\mathbf{C}^{d}\right) .
$$

It follows that the Bargmann transform is a homeomorphism from the Gelfand tripples in (2.9) and (2.10) to the Gelfand tripples

$$
\left(\mathcal{A}_{s}\left(\mathbf{C}^{d}\right), A^{2}\left(\mathbf{C}^{d}\right), \mathcal{A}_{s}^{\prime}\left(\mathbf{C}^{d}\right)\right) \quad \text { and } \quad\left(\mathcal{A}_{0, s}\left(\mathbf{C}^{d}\right), A^{2}\left(\mathbf{C}^{d}\right), \mathcal{A}_{0, s}^{\prime}\left(\mathbf{C}^{d}\right)\right),
$$

respectively. Furthermore, the definition of the spaces in Remark 4.4 agrees with corresponding spaces in (4.2) (cf. [16]).

It now follows that Theorems $1.3,4.3$ and $4.3^{\prime}$ remains valid after the spaces in (4.3) are replaced by corresponding spaces in (4.2), where the $L^{2}$ products are replaced by the $A^{2}$ products.

Acknowledgements Open access funding provided by Linnaeus University.

\section{Compliance with ethical standards}

Conflict of interest The author declares that he has no conflict of interest.

Human participants This article does not contain any studies with human participants or animals performed by the author.

Open Access This article is distributed under the terms of the Creative Commons Attribution 4.0 International License (http://creativecommons.org/licenses/by/4.0/), which permits unrestricted use, distribution, and reproduction in any medium, provided you give appropriate credit to the original author(s) and the source, provide a link to the Creative Commons license, and indicate if changes were made.

\section{References}

1. Bargmann, V. 1961. On a Hilbert space of analytic functions and an associated integral transform. Communications on Pure and Applied Mathematics 14: 187-214.

2. Cappiello, M., and L. Rodino. 2006. SG-pseudodifferential operators and Gelfand-Shilov spaces. Rocky Mountain Journal of Mathematics 36: 1117-1148.

3. Cappiello, M., and J. Toft. 2017. Pseudo-differential operators in a Gelfand-Shilov setting. Mathematische Nachrichten 290: 738-755.

4. Chen, Y., J. Toft, and P. Wahlberg. 2019. The Weyl product on quasi-Banach modulation spaces. Bulletin of Mathematical Sciences 9: 1950018-1.

5. Chung, J., S.-Y. Chung, and D. Kim. 1996. Characterizations of the Gelfand-Shilov spaces via Fourier transforms. Proceedings of the American Mathematical Society 124: 2101-2108.

6. Fernandez, C., A. Galbis, and J. Toft. 2017. The Bargmann transform and powers of harmonic oscillator on Gelfand-Shilov subspaces. RACSAM 111: 1-13.

7. Garetto, C., and M. Ruzhansky. 2015. Hyperbolic second order equations with non-regular time dependent coefficients. Archive for Rational Mechanics and Analysis 217: 113-154.

8. Gelfand, I.M., and G.E. Shilov. 1968. Generalized functions, II-III. NewYork, London: Academic Press. 
9. Hörmander, L. 1983, 1985. The analysis of linear partial differential operators, vol. I-III. Berlin, Heidelberg, NewYork: Springer.

10. Lozanov Crvenković, Z., and D. Perišić. 2007. Hermite expansions of elements of Gelfand Shilov spaces in quasianalytic and non quasianalytic case. Novi Sad Journal of Mathematics 37: 129-147.

11. Pilipović, S. 1986. Generalization of Zemanian spaces of generalized functions which have orthonormal series expansions. SIAM Journal on Mathematical Analysis 17: 477-484.

12. Pilipović, S. 1986. Structural theorems for periodic ultradistributions. Proceedings of the American Mathematical Society 98: 261-266.

13. Pilipović, S. 1988. Tempered ultradistributions. Bollettino dell'UMI - Unione Matematica Italiana 7: 235-251.

14. Reed, M., and B. Simon. 1979. Methods of modern mathematical physics, vol. I, II. London, New York: Academic Press.

15. Toft, J. 2012. The Bargmann transform on modulation and Gelfand-Shilov spaces, with applications to Toeplitz and pseudo-differential operators. Journal of Pseudo-Differential Operators and Applications 3: 145-227.

16. Toft, J. 2017. Images of function and distribution spaces under the Bargmann transform. J. PseudoDiffer. Oper. Appl. 8: 83-139.

17. Treves, F. 1967. Topological vector spaces, distributions and kernels. New York-London: Academic Press.

Publisher's Note Springer Nature remains neutral with regard to jurisdictional claims in published maps and institutional affiliations. 